\title{
Disturbed theta and gamma coupling as a potential mechanism for visuospatial working memory dysfunction in people with schizophrenia
}

Peter A. Lynn ${ }^{1,2}$ and Scott R. Sponheim ${ }^{1,2^{*}}$

* Correspondence: sponh001@umn.edu ${ }^{1}$ Minneapolis VA Health Care System, 1 Veterans Drive, Minneapolis, MN 55417, USA ${ }^{2}$ University of Minnesota, Minneapolis, MN 55455, USA

\section{() Biomed Central}

\begin{abstract}
Working memory (WM) deficits have been repeatedly observed in people with schizophrenia (PSZ) and their unaffected biological relatives (REL). Given the apparent association with genetic liability for schizophrenia, WM deficits have been proposed as a potential endophenotype for the disorder. Abnormal neural responses during WM performance have likewise been observed in PSZ and REL and may reflect the expression of genetic liability for schizophrenia in brain function. Relatively recent investigations have examined the role of neural oscillatory activity during visuospatial WM function in healthy people, as well as dysfunction in psychopathology. This research was in part motivated by a neural model of WM proposed by Lisman and Idiart (Science 267:1512-1515, 1995) that delineated a mechanism for representing multiple stimuli within WM through systematic interactions between neural oscillations in the theta- and gamma- frequency ranges. Aberrant oscillatory activity in theta and gamma frequency ranges has since been proposed as a potential underlying factor in WM dysfunction in PSZ and REL. The experimental evidence derived from studies of healthy people that pertains to the theta-gamma model of WM is reviewed herein. Although scarce, direct examinations of theta-gamma interactions in PSZ and REL are likewise reviewed in addition to reports of separate deficits in theta and gamma frequencies observed in PSZ during WM. The implications of theta and gamma oscillatory deficits reported in PSZ are discussed in the context of the Lisman and Idiart (Science 267:1512-1515, 1995) model, as well as how these deficits may result in aberrant theta-gamma interactions that give rise to visuospatial WM dysfunction. Given evidence supporting the Lisman and Idiart (Science 267:1512-1515, 1995) model of theta-gamma interactions in WM and the lack of direct exploration of the model in schizophrenia, there is an imperative to carry out formal testing of theta-gamma interactions in PSZ and REL during WM.
\end{abstract}

Keywords: Working memory, Schizophrenia, Theta, Gamma, Cross-frequency coupling

\section{Background}

Visuospatial working memory (WM), comprised by the coordination of processing, maintaining, manipulating, and retrieving spatial and visual information within the brain [1], has been implicated in a variety of tasks necessary for the navigation of dayto-day life, including wayfinding [2] and visuomotor learning [3, 4]. Prominent WM

(c) The Author(s). 2016 Open Access This article is distributed under the terms of the Creative Commons Attribution 4.0 International License (http://creativecommons.org/licenses/by/4.0/), which permits unrestricted use, distribution, and reproduction in any medium, provided you give appropriate credit to the original author(s) and the source, provide a link to the Creative Commons license, and indicate if changes were made. The Creative Commons Public Domain Dedication waiver (http://creativecommons.org/ publicdomain/zero/1.0/) applies to the data made available in this article, unless otherwise stated. 
deficits have been demonstrated across multiple sensory modalities in people with schizophrenia (PSZ), including in the visuospatial realm ([5 reviewed in 6]). Furthermore, better visuospatial WM function in PSZ has been shown to predict better functional and social outcomes [7, 8]. Spatial WM deficits are likewise observed in unaffected first-degree relatives of PSZ [9-11], suggesting they may represent genetic liability for schizophrenia and thus constitute a potential endophenotype for the disorder [12-15].

Electroencephalography (EEG) has been widely used to investigate the neural underpinnings of WM processes, traditionally in the form of event-related potential (ERP) studies (reviewed in [16]). PSZ have been found to demonstrate deficits in ERPs elicited from WM tasks [17-19] and tasks probing sustained attention [20-24], among others; similar deficits have been observed in first-degree relatives of people with the disorder $[22,25,26]$. More recently, EEG research has examined oscillatory activity in the brain associated with WM. Oscillatory activity in the brain, which arises from the rhythmic activation of large numbers of synapses [27], is crucial to coordinated activity in the normally functioning brain [28]. PSZ demonstrate many abnormalities in oscillatory activity, which interfere with efficient integration of activity within the brain and may contribute to the prominent cognitive and functional deficits observed in the disorder [27-29].

Generally speaking, high frequency oscillations are associated with synchronization in local cortical networks, and lower frequency oscillations with synchronization between brain structures/across greater distances [28]. Oscillations in the gamma-band (25$100 \mathrm{~Hz}$ ) and the theta-band $(4-8 \mathrm{~Hz})$ in particular have been widely implicated in episodic memory and WM alike (reviewed in [30]). Furthermore, the synchronized superimposition of gamma cycles on oscillatory theta activity has been proposed as a mechanism for the representation of multiple items in WM, a mechanism termed the "theta/gamma code" [31]. Multi-item WM has been associated with phase-phase coupling (see Fig. 3) between theta and gamma frequencies in healthy people (e.g., [32]). In addition, theta-gamma phase-amplitude coupling (Fig. 3) has been observed in the hippocampus $[33,34]$ as well as from scalp EEG $[35,36]$ in healthy humans and, in both cases, has been predictive of WM performance [33-36]. Given these findings, abnormal theta-gamma coupling has been proposed as a mechanism for impaired WM function in PSZ [37, 38], though this hypothesis has yet to be formally investigated.

Much research has already shown abnormalities in the gamma- and theta- bands in PSZ, suggesting dysfunction in neural integration on local and global scales. The present review examines gamma and theta abnormalities during WM in PSZ in the context of the theta/gamma neural code. Though the theta/gamma code has not been formally investigated in schizophrenia to date, the mechanism and hypothesized utility of the superimposition of gamma cycles on theta cycles in relation to WM in healthy people will be reviewed. Subsequently, this review will recount observed abnormalities in PSZ for activity in the gamma-band, theta-band, and interactions between the two during WM, discussing how such abnormalities may interfere with efficient theta/ gamma coding in schizophrenia. Through integration of current findings regarding abnormalities in gamma- and theta-band oscillations in schizophrenia during WM, the present review will attempt to elucidate the potential implications of these abnormalities with respect to the theta/gamma neural code. 
The superimposition of gamma-band oscillations on theta oscillations: the theta/gamma neural code

The theory of a theta/gamma code as first posited by Lisman and Idiart [39] and elaborated elsewhere (e.g., [40]) offered three primary, interrelated proposals: 1) that systematic coupling of oscillatory activity of different frequencies within the brain is meaningful, 2) that this coupling serves to form a temporal code in which distinct pieces of information are associated with simultaneous activity across several cells at particular phases within a cycle of theta, and 3) that the theta/gamma code model can explain central features of WM. In an effort to support their first two hypotheses regarding the importance of cross-frequency coupling as a temporal code, the authors first addressed the plausibility of oscillatory activity as a sustained temporal organizer. The neuromodulators acetylcholine and serotonin have been shown to be released in the brain during periods of oscillatory activity $[41,42]$. In the presence of these neuromodulators, firing of neuronal cells induces a period of membrane afterdepolarization (ADP) rather than the typical afterhyperpolarization, leading to a transient increase in cell excitability [43-45]. Building off these findings, the authors used computer simulations to show that the duration of ADP was on the time scale of oscillations in the alpha-theta range (5 to $12 \mathrm{~Hz}$ ), and that such ADP could be propagated for many cycles. Thus, a single excitatory input could lead to sustained firing on subsequent oscillatory cycles, potentially serving a storage function (Fig. 1a; [39]). Furthermore, the authors noted that in Sternberg's [46] classic work on serial scanning in WM, the addition of each stimulus to the string of stimuli to be recalled resulted in an increase in reaction time of roughly $38 \mathrm{~ms}-$ an increase corresponding to the cycle of a neural oscillation in the beta-gamma range. Jensen and Lisman [47] elaborated on this observation, showing that the theta-gamma code model could effectively account for reaction time data and serial position effects reported by Sternberg $[46,48] .{ }^{1}$ Roughly seven cycles in the beta-gamma range could be superimposed on a lower frequency cycle in the alpha-theta range-such as those cycles induced by the ADP-which Lisman and Idiart [39] emphasized corresponded to the canonical average capacity of WM determined by Miller [49]. Thus, Lisman and Idiart [39] demonstrated the potential for theta-gamma cross-frequency coupling to serve an ongoing, organizing function.

In their discussion of the temporal properties of theta and gamma oscillations in relation to Sternberg's classic studies [46, 48], Lisman and Idiart [39] transition to their final proposal regarding the role of the theta/gamma code in WM. The authors demonstrated through simulation how differing, non-overlapping stimuli or "memories" ([39], p. 1515) could be stored through systematic variation in the phases of the different high-frequency subcycles, with each stimulus being represented by different groups of cells that maintain the stimulus by firing simultaneously at a particular high-frequency subcycle within the nesting lower frequency cycle (Fig. 1c). Thus, particular stimuli or memories are represented by the particular spatial pattern of a group of cells, or neural ensemble [37], these memories are propagated or maintained through interactions between oscillatory activity in the theta and gamma frequencies, and sequential information regarding these memories are linked to the particular gamma subcycle within the nesting theta cycle a given memory's neural ensemble produces (or perhaps more accurately, the phase offset between the neural ensemble's gamma cycle and the nesting theta cycle; $[37,39])$. The theta-gamma neural code as proposed by Lisman and 


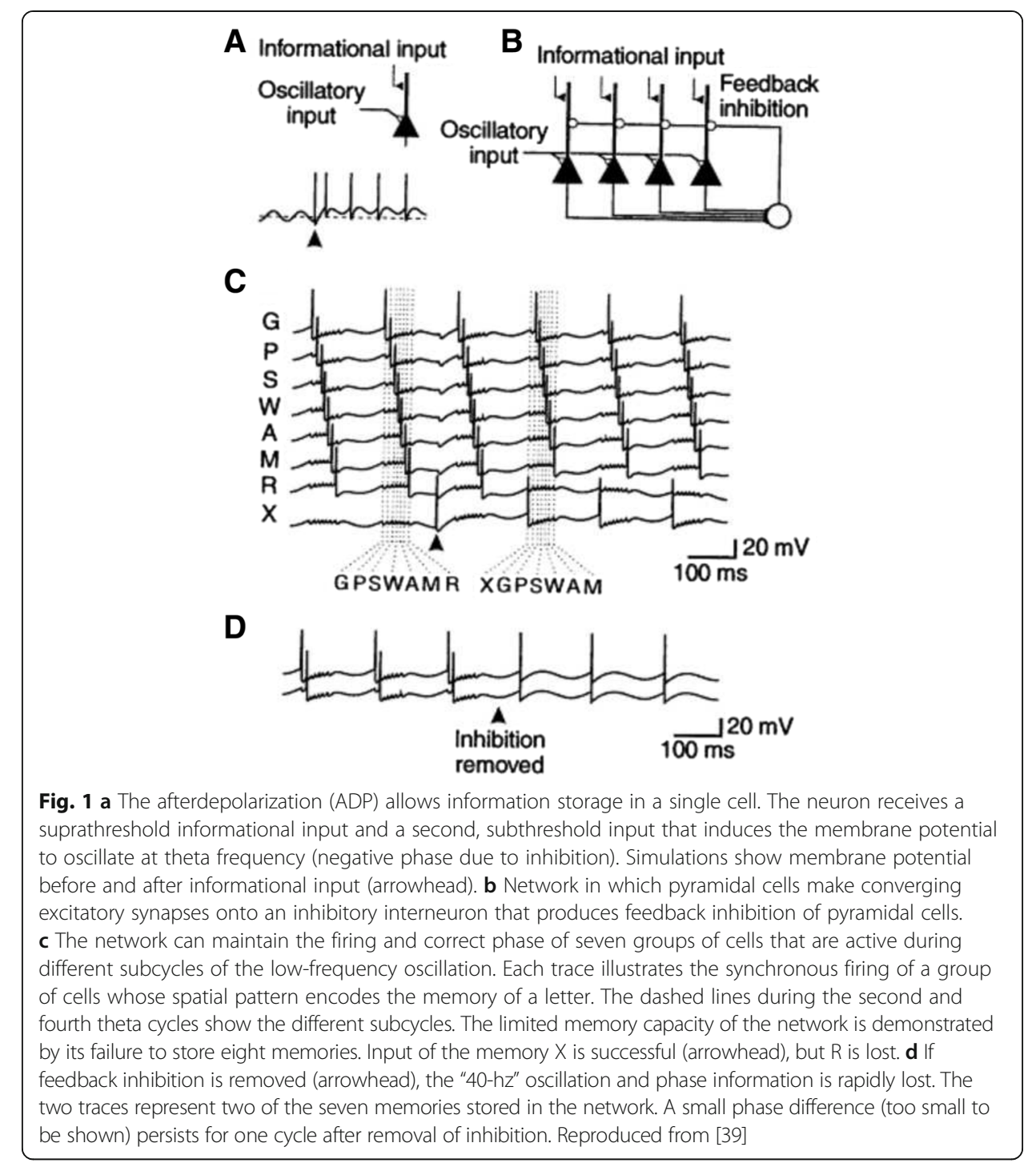

Idiart [39] represents a theoretical framework which, at its inception, integrated a number of experimental findings to provide a plausible model for the storage of multiple items in WM. This framework, which has since been further developed [e.g., $31,37]$, has served as the basis for a substantial body of experimentation over the past twenty years, the vast majority of which has produced results supporting the Lisman and Idiart [39] model. A brief review of this sizeable body of literature follows, focused on studies probing WM function.

\section{Empirical support for the theta-gamma neural code}

The experimental support for Lisman and Idiart's [39] model of the theta-gamma neural code is now widespread. In addition to other supporting observations, Lisman and Idiart [39] cited as some of the most significant early support for the theta-gamma neural code model the work of O'Keefe and Recce [50] (later clarified by [51]). These researchers examined pyramidal cells from the hippocampus of rats known as place 
cells, which fire bursts of spikes as the animal moves through a particular area in its environment termed the place field of that cell [50]. Concurrent to these bursts, theta band activity also occurs in the hippocampal EEG as a rat relocates itself in its environment. Though phase correlations between theta-band activity and bursts of hippocampal activity had then been well-established [52-55], the nature of the relationship was not well clarified. Using a combination of single unit and EEG recordings from hippocampal CA1 and CA3 place cells, O'Keefe and Recce [50] showed that for a single run through the field of a given place cell on a linear track, the cell fired bursts of spikes at progressively earlier parts of the of the concomitant theta cycle, a phenomenon termed "phase precession" ([51], p. 149; Fig. 2). This finding was taken to indicate that the hippocampus may use a neural code in which theta phase conveys important information [31] - namely, the theta-gamma neural code that Lisman and Idiart [39] proposed. Skaggs and colleagues [51] replicated and elaborated this finding to demonstrate that the phase precession effect was indeed robust throughout the rat hippocampus, and observed as animals moved through two-dimensional in addition to one-dimensional space. More significantly, the researchers emphasize the support their findings lend to the notion that the theta phase-offset at which a place cell fires carries important spatial information [51]. A considerable body of work has shown that this spiking in the hippocampus and entorhinal cortex is time locked to local gamma-activity, such that firing is effectively limited to discrete, gamma-locked time windows (see [56] and [57]). Bieri, Bobbitt, and Colgin [58] similarly observed theta-gamma phase precession in rat CA1 hippocampal cells and suggested differential relationships between coupling between high- and low-gamma signals. Phase precession has likewise been observed in entorhinal grid cells [59], supporting the organization of multi-item WM by way of theta- and gamma- cycles as by Lisman and Idiart's [39] model and its elaborations. Phase-precession is further reviewed in [56].

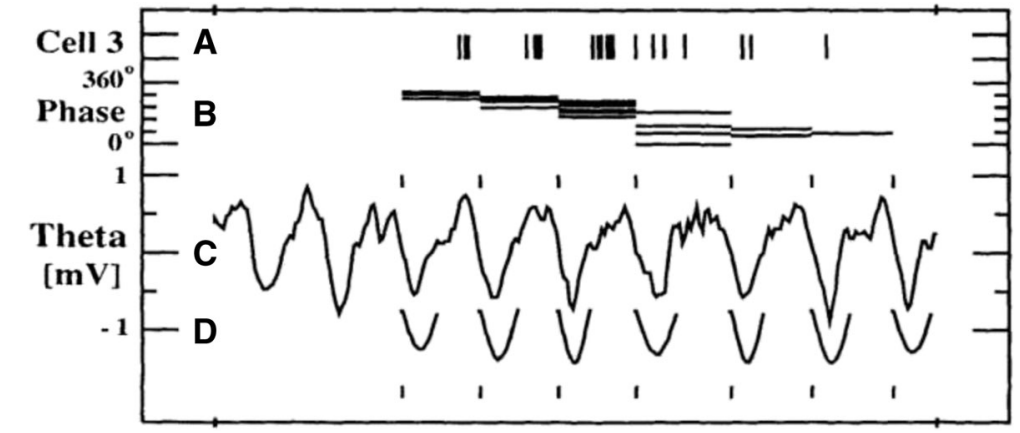

Time [s]

Fig. 2 Extraction of the firing phase shift for each spike during a single run through the field of a CA3 place cell on the linear track. a Each firing of cell 3 as a single vertical line during the $1 \mathrm{~s}$ of data. $\mathbf{b}$ Phase of each spike relative to the theta cycle within which it falls as a horizontal line. c Hippocampal theta activity recorded at the same time as the single unit. $\mathbf{d}$ Result of the theta template matching algorithm. Note that the amplitude and the time between onsets of each template match varies to fit the variations in the theta. The small vertical ticks above the electroencephalogram (EEG) and below the template fits mark the beginning of each theta cycle. The cell clearly fires six bursts of spikes during this run through the place field. Comparison of each burst with the concomitant theta wave shows that each successive burst fires on an earlier part of the theta. This is shown clearly by the descending staircase of the phase correlates in B. EEG voltage in $C$ is +1 to $-1 \mathrm{mV}$. Total time between marks on the $x$ axis is $1 \mathrm{~s}$. Reproduced from [50] 
Much subsequent experimentation has added to the support for Lisman and Idiart's [39] model, demonstrating systematic relationships between theta- and gamma- oscillatory activity. These relationships are most frequently presented in one of two forms: phase-phase coupling (also called phase-synchrony, or simply phase-coupling), in which a given phase angle of a cycle of ongoing theta-activity tends to occur at a given phase angle of concurrent gamma-activity; and phase-amplitude coupling, in which the amplitude of gamma activity is modulated by the phase of concurrent theta-oscillations (Fig. 3). Though these types of coupling are most frequently reported separately, it has been suggested that they are likely two expressions of a single phenomenon $[60,61]$.

Findings of both types of coupling are summarized in Tables 1 and 2. Review of the animal studies in Table 1 reveals well-documented increases of theta-gamma coupling using intracranial recordings from the entorhinal-hippocampal system of rodents during performance of memory-focused tasks (e.g., Table 1, references i-xi). Thetagamma coupling is likewise observed using intracranial recordings from the entorhinal cortex and hippocampus during free exploration, sleep and anesthetization (e.g., xiixxvi) as well as from in vitro isolated samples (e.g., xxvii-xxix) in rats and mice. Primate studies have also revealed theta-gamma coupling in auditory cortex $(\mathrm{xxx})$ during a passive listening task as well as from anterior cingulate cortex during an attention task (xxxi). Thus, WM processes are associated with increased theta-gamma coupling in the animal literature, though coupling observed in less-memory-dependent paradigms indicates that theta-gamma coupling may represent a more widespread means of communication in the brains of rodents and primates.

Table 2 reports findings of theta-gamma coupling in humans. Here, we see intracranial recordings utilized to demonstrate phase-phase coupling (e.g., Table 2, references i, vii) as well as phase-amplitude coupling (e.g., ii-vi) between theta- and gamma- activity in hippocampal areas as well as throughout the cortex. Furthermore, scalp-level recordings have shown phase-phase theta-gamma coupling in humans over primarily frontal, parietal and occipital areas (e.g., viii-xi, xv), as well as phase-amplitude coupling over much of the scalp, including prefrontal, occipital and parietal sites (e.g. xi, xiii, xiv). Notably, one recent experiment temporarily increased individuals' short-term memory capacities by using transcranial alternating current stimulation to slow their theta

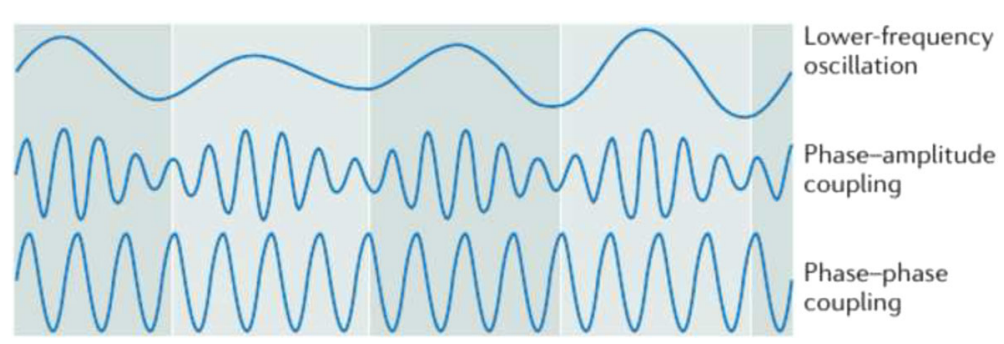

Fig. 3 Schematic overview of two cases in which oscillatory activity of a higher-frequency oscillation may be related to the phase of a lower-frequency oscillation. Dark and light boxes separate consecutive cycles of the lower-frequency oscillation. The lower-frequency oscillation of fluctuating amplitude (top) shows phase-amplitude coupling with a higher-frequency oscillation of fluctuating amplitude (middle). In this example, amplitudes of the higher-frequency oscillation are maximal during the up-phase of the lowerfrequency oscillation. A higher-frequency oscillation of stable amplitude (bottom), shows phase-phase coupling with the low-frequency oscillation. Here, peaks of the higher-frequency oscillation always coincide with the same phase values of the lower-frequency oscillations. Reproduced from [79] 
Table 1 Animal findings regarding theta-gamma coupling (TGC)

\begin{tabular}{|c|c|c|c|c|c|c|}
\hline $\begin{array}{l}\text { Authors \& Year of } \\
\text { publication }\end{array}$ & Subjects & Measure & Region of brain examined & $\begin{array}{l}\text { Task performed / } \\
\text { behavior during } \\
\text { measurement }\end{array}$ & Relevant findings & Conclusions \\
\hline \multicolumn{7}{|c|}{ Studies featuring memory-related tasks } \\
\hline $\begin{array}{l}\text { i. Tort et al. } \\
\text { (2008) [80] }\end{array}$ & $\begin{array}{l}6 \text { male Sprague- } \\
\text { Dawley rats }\end{array}$ & iEEG & $\begin{array}{l}\text { Striatum, } \\
\text { hippocampus }\end{array}$ & T-maze & $\begin{array}{l}\text { - Theta phase modulated high } \\
\text { frequency }(80-120 \mathrm{~Hz}) \text { but not low } \\
\text { frequency }(30-60 \mathrm{~Hz}) \text { gamma in } \\
\text { striatum } \\
\text { - TGC between low and high gamma } \\
\text { power and theta phase in hippocampus } \\
\text { - TGC strongest during decision making } \\
\text { portions of task } \\
\text { - Striatal theta phase modulated } \\
\text { hippocampal high gamma power }\end{array}$ & $\begin{array}{l}\text { - Low and high frequency } \\
\text { gamma may represent } \\
\text { distinct physiological } \\
\text { processes } \\
\text { - TGC phase-amplitude } \\
\text { coupling likely related to } \\
\text { engagement of cognitive } \\
\text { processes across varying } \\
\text { time scales }\end{array}$ \\
\hline $\begin{array}{l}\text { ii. Tort, Komorowski, } \\
\text { Manns, Kopell, \& } \\
\text { Eichenbaum } \\
\text { (2009) }[81]\end{array}$ & 6 male Long-Evans rats & iEEG & $\begin{array}{l}\text { Hippocampus } \\
\text { (CA3) }\end{array}$ & $\begin{array}{l}\text { - Freely behaving } \\
\text { - Item-context } \\
\text { learning task }\end{array}$ & $\begin{array}{l}\text { - Theta phase modulated low gamma } \\
\text { power in CA3 during free behavior } \\
\text { - TGC in CA3 increased during learning } \\
\text { and remained high during } \\
\text { overtraining sessions } \\
\text { - Strength of TGC predicted mean } \\
\text { performance accuracy }\end{array}$ & $\begin{array}{l}\text { TGC important to memory } \\
\text { processing }\end{array}$ \\
\hline $\begin{array}{l}\text { iii. Shirvalkar, Rapp, } \\
\text { \& Shapiro } \\
\text { (2010) [82] }\end{array}$ & 6 male Long-Evans rats & iEEG & Hippocampus & $\begin{array}{l}\text { Matching-to-place } \\
\text { task (six-arm radial } \\
\text { water maze) }\end{array}$ & $\begin{array}{l}\text { - Power-power TGC increased during } \\
\text { retrieval as compared to exploration } \\
\text { (encoding) } \\
\text { - Power-power TGC higher for } \\
\text { successful versus unsuccessful recall } \\
\text { - Strength of TGC predicted memory } \\
\text { performance, while indices of theta } \\
\text { or gamma power alone did not }\end{array}$ & $\begin{array}{l}\text { Power-power TGC in } \\
\text { hippocampus } \\
\text { is important to memory- } \\
\text { dependent behavior }\end{array}$ \\
\hline $\begin{array}{l}\text { iv. Belluscio, } \\
\text { Mizuseki, Schmidt, } \\
\text { Kempter, \& Buzsáki } \\
\text { (2012) [60] }\end{array}$ & 6 male Long-Evans rats & iEEG & $\begin{array}{l}\text { Hippocampus } \\
\text { (CA1 pyramidal } \\
\text { cells) }\end{array}$ & $\begin{array}{l}\text { - Maze exploration } \\
\text { - REM sleep }\end{array}$ & $\begin{array}{l}\text { - Phase-phase and phase- } \\
\text { amplitude TGC }\end{array}$ & $\begin{array}{l}\text { TGC aids in coordinating } \\
\text { neuronal spiking across } \\
\text { multiple time scales, } \\
\text { potentially helpful in transfer } \\
\text { of information and plasticity } \\
\text { dependent upon spike timing }\end{array}$ \\
\hline
\end{tabular}


Table 1 Animal findings regarding theta-gamma coupling (TGC) (Continued)

\begin{tabular}{|c|c|c|c|c|c|c|}
\hline $\begin{array}{l}\text { v. Cabral et al. } \\
\text { (2014) [83] }\end{array}$ & $\begin{array}{l}8 \text { NR1-KO mice ("knockouts" } \\
\text { lacking NR1 NMDAR subunit } \\
\text { in principal CA1 neurons), } \\
7 \text { littermate controls }\end{array}$ & $\mathrm{iEEG}$ & $\begin{array}{l}\text { Hippocampus } \\
\text { (dorsal CA1) }\end{array}$ & Five-armed "starmaze" & $\begin{array}{l}\text { - Control mice showed increased TGC } \\
\text { between theta phase and high-gamma } \\
\text { amplitude for place-strategy/allocentric } \\
\text { trials and increased TGC between theta } \\
\text { phase and low-gamma amplitude } \\
\text { during sequence-strategy/ } \\
\text { egocentric trials } \\
\text { - Excess high and low gamma } \\
\text { observed in knockout mice }\end{array}$ & $\begin{array}{l}\text { - Preferred frequency of } \\
\text { gamma in TGC associated } \\
\text { with spatial WM } \\
\text { dependent on strategy used } \\
\text { - Dynamic strategy switching } \\
\text { is disrupted by NMDAR } \\
\text { disruption }\end{array}$ \\
\hline $\begin{array}{l}\text { vi. Igarashi, Lu, } \\
\text { Colgin, Moser, } \\
\& \text { Moser } \\
\text { (2014) [84] }\end{array}$ & 17 male Long-Evans rats & iEEG & $\begin{array}{l}\text { Entorhinal cortex } \\
\text { (medial and lateral, layer III), } \\
\text { hippocampus (CA1) }\end{array}$ & $\begin{array}{l}\text { Odor-place } \\
\text { association task }\end{array}$ & $\begin{array}{l}\text { - Odor-place learning was accompanied } \\
\text { by increased phase-amplitude TGC in CA1 } \\
\text { during odor-sampling (retrieval) } \\
\text { - Phase-amplitude TGC was observed in } \\
\text { lateral EC from beginning of task } \\
\text { - Gamma (20-40Hz) power in both regions } \\
\text { was unaltered }\end{array}$ & $\begin{array}{l}\text { - TGC in hippocampus } \\
\text { important to retrieval } \\
\text { of learned memories } \\
\text { - Learning associated with } \\
\text { more extensive coupling } \\
\text { of already- } \\
\text { existing } \\
\text { gamma rhythms }\end{array}$ \\
\hline $\begin{array}{l}\text { vii. Nishida, } \\
\text { Takahashi, } \\
\text { \& Lauwereyns } \\
\text { (2014) [85] }\end{array}$ & 4 male Wistar/ST rats & $\mathrm{iEEG}$ & $\begin{array}{l}\text { Hippocampus } \\
\text { (CA1) }\end{array}$ & $\begin{array}{l}\text { Memory-guided } \\
\text { spatial alternation } \\
\text { task }\end{array}$ & $\begin{array}{l}\text { Modulation of gamma-activity by theta } \\
\text { phase strengthened overall from } \\
\text { beginning to end of session }\end{array}$ & $\begin{array}{l}\text { Increase in TGC may reflect } \\
\text { plasticity of CA1-CA3/C } \\
\text { A1-EC network, suggestive of } \\
\text { optimized communication } \\
\text { between these areas }\end{array}$ \\
\hline $\begin{array}{l}\text { xiii. Schomburg } \\
\text { et al. (2014) [57] }\end{array}$ & $\begin{array}{l}9 \text { male Long-Evans, } \\
3 \text { male Sprague-- } \\
\text { Dawley rats }\end{array}$ & $\mathrm{iEEG}$ & $\begin{array}{l}\text { Hippocampus, } \\
\text { entorhinal cortex }\end{array}$ & $\begin{array}{l}\text { - Linear track } \\
\text { - T-maze } \\
\text { - Open field } \\
\text { - REM Sleep }\end{array}$ & $\begin{array}{l}\text { - Strong TGC between theta phase and } \\
\text { gamma power in hippocampus during } \\
\text { memory recall } \\
\text { - Gamma amplitude also modulated by } \\
\text { theta phase in EC } \\
\text { - Preferred theta phase dependent upon } \\
\text { from where input is being received }\end{array}$ & $\begin{array}{l}\text { Temporal coordination of } \\
\text { activity in entorhinal- } \\
\text { hippocampus complex } \\
\text { primarily supported by } \\
\text { theta- and low-frequency } \\
\text { gamma activity }\end{array}$ \\
\hline
\end{tabular}


Table 1 Animal findings regarding theta-gamma coupling (TGC) (Continued)

\begin{tabular}{|c|c|c|c|c|c|c|}
\hline $\begin{array}{l}\text { ix. Takahashi, } \\
\text { Nishida, Redish, } \\
\text { \& Lauwereyns } \\
\text { (2014) [86] }\end{array}$ & 4 male Wistar/ST rats & $\mathrm{iEEG}$ & $\begin{array}{l}\text { Hippocampus } \\
\text { (CA1) }\end{array}$ & $\begin{array}{l}\text { Memory-guided } \\
\text { spatial alternation } \\
\text { task }\end{array}$ & $\begin{array}{l}\text { Gamma-amplitudes in CA1 were } \\
\text { phase-locked to theta during a "fixation" } \\
\text { period prior to task performance } \\
\text { - Preferred theta-phase differed between } \\
\text { high- }(60-90 \mathrm{~Hz}) \text { and low- }(30-45 \mathrm{~Hz}) \\
\text { gamma } \\
\text { - low-gamma activity increased with a } \\
\text { concurrent decrease in high-gamma } \\
\text { activity towards the end of the } \\
\text { fixation period }\end{array}$ & $\begin{array}{l}\text { High-gamma activity } \\
\text { associated with externally } \\
\text { cued information } \\
\text { processing, low-gamma with } \\
\text { internally generated } \\
\text { information processing }\end{array}$ \\
\hline $\begin{array}{l}\text { X. Trimper, } \\
\text { Stefanescu, } \\
\text { \& Manns } \\
\text { (2014) [87] }\end{array}$ & 6 male Long-Evans rats & iEEG & Entorhinal cortex & $\begin{array}{l}\text { Novel object } \\
\text { recognition } \\
\text { memory task }\end{array}$ & $\begin{array}{l}\text { - Increased theta-high-gamma } \\
\text { phase-amplitude coupling in the } \\
\text { hippocampi of rats exploring } \\
\text { novel objects } \\
\text { - Gamma-gamma phase synchrony } \\
\text { between CA3 and CA1 LFPs that } \\
\text { varied with relative theta- phase } \\
\text { and was greatest for objects } \\
\text { subsequently remembered }\end{array}$ & $\begin{array}{l}\text { TGC associated with memory } \\
\text { processing, but differentially } \\
\text { dependent on frequency of } \\
\text { gamma activity }\end{array}$ \\
\hline $\begin{array}{l}\text { xi. Siegle \& Wilson } \\
\text { (2014) [88] }\end{array}$ & $\begin{array}{l}\text { Male parvalbumin-Cre } \\
\text { (PV-Cre) heterozygote mice }\end{array}$ & iEEG & Hippocampus & T-maze & $\begin{array}{l}\text { - High-gamma modulated by } \\
\text { theta-phase during T-maze } \\
\text { performance } \\
\text { - Optogenetic stimulation of } \\
\text { inhibitory interneurons at } \\
\text { trough of theta improved } \\
\text { task performance during retrieval, } \\
\text { while stimulation at theta peaks } \\
\text { improved performance } \\
\text { during encoding }\end{array}$ & $\begin{array}{l}\text { - Encoding and retrieval } \\
\text { processes occur at } \\
\text { different preferential } \\
\text { theta phases } \\
\text { - Phase-specific inhibition may } \\
\text { reduce the response to } \\
\text { task-irrelevant inputs }\end{array}$ \\
\hline \multicolumn{7}{|c|}{ Studies featuring free exploration, sleep or anesthetization } \\
\hline $\begin{array}{l}\text { xii. Buzsáki, Leung, } \\
\& \text { Vanderwolf } \\
\text { (1983) [53] }\end{array}$ & 43 male Long Evans rats & iEEG & Hippocampus & $\begin{array}{l}\text { - Activity wheel } \\
\text { - Immobility }\end{array}$ & $\begin{array}{l}\text { - Fast EEG (gamma: } 25-70 \mathrm{~Hz} \text { ) as } \\
\text { well as interneuron spiking } \\
\text { superimposed upon } \\
\text { and modulated by theta phase } \\
\text { - More prominent in activity than } \\
\text { immobility }\end{array}$ & $\begin{array}{l}\text { Slow activity (theta) may be } \\
\text { generated through feed-forward } \\
\text { inhibition from septum and } \\
\text { direct excitation from } \\
\text { entorhinal cortex }\end{array}$ \\
\hline
\end{tabular}


Table 1 Animal findings regarding theta-gamma coupling (TGC) (Continued)

\begin{tabular}{|c|c|c|c|c|c|c|}
\hline $\begin{array}{l}\text { xiii. Soltesz } \\
\text { \& Deschenes } \\
\text { (1993) [89] }\end{array}$ & $\begin{array}{l}\text { Male and female Sprague } \\
\text { Dawley rats }\end{array}$ & $\mathrm{iEEG}$ & $\begin{array}{l}\text { Hippocampus } \\
\text { (CA1, CA3) }\end{array}$ & $\begin{array}{l}\text { Ketamine-xylazine } \\
\text { anesthesia }\end{array}$ & $\begin{array}{l}\text { - Injection of } \mathrm{Cl}^{-} \text {ions into pyramidal } \\
\text { cells brought on high frequency } \\
\text { ( } 25-50 \mathrm{~Hz} \text { ) Oscillation modulated } \\
\text { at theta-frequency }\end{array}$ & $\begin{array}{l}\text { - Fast oscillations generated } \\
\text { by } \mathrm{Cl}^{-} \text {dependent } \mathrm{GABA}_{\mathrm{A}} \\
\text { receptors } \\
\text { - Theta modulation of fast } \\
\text { oscillation in hippocampus } \\
\text { likely arises through } \\
\text { interaction between } \\
\text { cholinergic and GABAergic } \\
\text { neurotransmitter systems }\end{array}$ \\
\hline $\begin{array}{l}\text { xiv. Bragin et al. } \\
\text { (1995) [90] }\end{array}$ & $\begin{array}{l}45 \text { male and female } \\
\text { Sprague-Dawley rats }\end{array}$ & $\mathrm{iEEG}$ & $\begin{array}{l}\text { Hippocampus } \\
\text { (dentate hilus) }\end{array}$ & $\begin{array}{l}\text { - Freely behaving } \\
\text { - Immobility } \\
\text { - REM Sleep }\end{array}$ & $\begin{array}{l}\text { - Prominent theta-phase to } \\
\text { gamma-amplitude coupling, } \\
\text { particularly in dentate hilus region, } \\
\text { during activity and REM sleep }\end{array}$ & $\begin{array}{l}\text { - TGC due to reciprocal } \\
\text { connections between } \\
\text { interneurons, hilar mossy } \\
\text { cells and CA3 pyramidal cells }\end{array}$ \\
\hline $\begin{array}{l}\text { xv. Chrobak \& } \\
\text { Buzsáki (1998) [91] }\end{array}$ & 19 Sprague Dawley rats & $\mathrm{iEEG}$ & $\begin{array}{l}\text { Entorhinal cortex } \\
\text { (layers II \& III), } \\
\text { Hippocampus }\end{array}$ & Freely behaving & $\begin{array}{l}\text { - Nesting of gamma oscillations within } \\
\text { theta oscillations in the entorhinal cortex } \\
\text { and hippocampus } \\
\text { - Neuronal spiking in entorhinal cortex } \\
\text { in phase with the local, nested } \\
\text { gamma oscillations } \\
\text { - Synchronization between theta-gamma } \\
\text { rhythms in entorhinal cortex and dentate } \\
\text { hilar region of hippocampus }\end{array}$ & $\begin{array}{l}\text { Systematic phase-locking of } \\
\text { gamma oscillations to nesting } \\
\text { theta oscillations is necessary } \\
\text { for communication within } \\
\text { perforant pathway }\end{array}$ \\
\hline $\begin{array}{l}\text { xvi. Buzsáki et al. } \\
\text { (2003) [92] }\end{array}$ & $\begin{array}{l}13 \text { hybrid (C57B6/J \& } \\
129 S 6 / \text { SVEVTac) and } 3 \\
\text { inbred (C57B6/J) mice }\end{array}$ & $\mathrm{iEEG}$ & $\begin{array}{l}\text { Hippocampus } \\
\text { (CA1 pyramidal } \\
\text { layer, dentate gyrus) }\end{array}$ & $\begin{array}{l}\text { - Freely behaving } \\
\text { - Immobile awake } \\
\text { - Sleeping }\end{array}$ & $\begin{array}{l}\text { Gamma, interneurons and pyramidal } \\
\text { cells all phase-locked to concurrent } \\
\text { theta rhythm during free behavior }\end{array}$ & $\begin{array}{l}\text { - Mouse brain is similar to } \\
\text { rat brain } \\
\text { - Interneurons critical to } \\
\text { gamma generation }\end{array}$ \\
\hline $\begin{array}{l}\text { xvii. Csicsvari, } \\
\text { Jamieson, Wise, } \\
\text { \& Buzsáki } \\
\text { (2003) [93] }\end{array}$ & $\begin{array}{l}12 \text { male Sprague- } \\
\text { Dawley rats }\end{array}$ & $\mathrm{iEEG}$ & $\begin{array}{l}\text { Hippocampus } \\
\text { (CA1, CA3, granule cell } \\
\text { layers) }\end{array}$ & $\begin{array}{l}\text { - Freely behaving } \\
\text { - Immobility } \\
\text { - Slow wave sleep } \\
\text { - REM sleep }\end{array}$ & $\begin{array}{l}\text { - Gamma power varied with theta phase } \\
\text { when theta present but irregularly } \\
\text { otherwise } \\
\text { - Gamma field power greater in } \\
\text { CA1 during theta-associated behaviors } \\
\text { - Gamma CSD power greater in granule } \\
\text { cell layer during theta-associated } \\
\text { behaviors }\end{array}$ & $\begin{array}{l}\text { Concurrent theta is not } \\
\text { necessary for gamma } \\
\text { oscillation, but theta } \\
\text { enhances and modulates } \\
\text { gamma when present }\end{array}$ \\
\hline
\end{tabular}


Table 1 Animal findings regarding theta-gamma coupling (TGC) (Continued)

\begin{tabular}{|c|c|c|c|c|c|c|}
\hline $\begin{array}{l}\text { xviii. Hentschke, } \\
\text { Perkins, Pearce, } \\
\text { \& Banks (2007) [94] }\end{array}$ & B6129SF2/J wild-type mice & iEEG & $\begin{array}{l}\text { Hippocampus } \\
\text { (CA1, all laminae) }\end{array}$ & $\begin{array}{l}\text { - Freely behaving } \\
\text { - Immobile awake }\end{array}$ & $\begin{array}{l}\text { - Phase-amplitude TGC during } \\
\text { exploration and immobility, highest } \\
\text { around hippocampal fissure } \\
\text { - Significantly decreased by injection } \\
\text { of atropine, a muscarinic antagonist }\end{array}$ & $\begin{array}{l}\text { Phase-amplitude TGC in CA1 } \\
\text { influenced by neurons with } \\
\text { muscarinic receptors }\end{array}$ \\
\hline $\begin{array}{l}\text { xix. Sirota et al. } \\
\text { (2008) [95] }\end{array}$ & 28 rats, 11 mice & iEEG & $\begin{array}{l}\text { Hippocampus, } \\
\text { neocortex }\end{array}$ & $\begin{array}{l}\text { - Active }(n=28 \\
\text { rats, } 11 \text { mice }) \\
\text { - Anesthetized } \\
(n=27 \text { rats })\end{array}$ & $\begin{array}{l}\text { - Phase-phase coupling between } \\
\text { hippocampal theta rhythms and } \\
\text { gamma oscillations in multiple } \\
\text { regions of neocortex, including } \\
\text { prefrontal cortex and primary } \\
\text { sensory areas }\end{array}$ & $\begin{array}{l}\text { TGC between hippocampus } \\
\text { and neocortex means for } \\
\text { transfer of information from } \\
\text { neocortex to hippocampus }\end{array}$ \\
\hline $\begin{array}{l}\text { xx. Wulff et al. } \\
\text { (2009) [96] }\end{array}$ & $\begin{array}{l}\text { PV- } \Delta Y 2 \text { mice (mice with } \\
\text { GABA }_{A} \text { receptor } \gamma 2 \text { subunit } \\
\text { ablated from parvalbumin- } \\
\text { positive interneurons in } \\
\text { hippocampus); normal } \\
\text { litter-mates as controls }\end{array}$ & iEEG & Hippocampus & Freely behaving & $\begin{array}{l}\text { - Phase-amplitude TGC nearly } \\
\text { three times as weak in PV- } \Delta Y 2 \\
\text { as control mice }\end{array}$ & $\begin{array}{l}\mathrm{PV}+\text { neurons involved in } \\
\text { coupling of theta to gamma } \\
\text { activity }\end{array}$ \\
\hline $\begin{array}{l}\text { xxi. Quilichini, } \\
\text { Sirota, \& Buzsáki } \\
\text { (2010) [97] }\end{array}$ & $\begin{array}{l}39 \text { male Sprague- } \\
\text { Dawley rats }\end{array}$ & $\mathrm{iEEG}$ & $\begin{array}{l}\text { Entorhinal cortex } \\
\text { (layers II, III, V) }\end{array}$ & - Anesthetized & $\begin{array}{l}\text { - Gamma (including high frequency) } \\
\text { power modulated by theta phase } \\
\text { in all layers } \\
\text { - Different theta phase preferences } \\
\text { from layer to layer }\end{array}$ & $\begin{array}{l}\text { Gamma activity can be } \\
\text { generated locally in individual } \\
\text { EC layers and relate to phase } \\
\text { of hippocampal theta activity }\end{array}$ \\
\hline $\begin{array}{l}\text { xxii. De Almeida, } \\
\text { Idiart, Villavicencio, } \\
\text { \& Lisman (2012) [59] }\end{array}$ & Rats & iEEG & $\begin{array}{l}\text { Entorhinal cortex } \\
\text { (grid cells) }\end{array}$ & $\begin{array}{l}\text { - Open field exploration } \\
\text { - Traversal of } \\
\text { linear track }\end{array}$ & $\begin{array}{l}\text { - Phase precession observed in grid } \\
\text { cells with two varying "modes": } \\
\text { inbound (firing occurs as rat } \\
\text { approaches center of place field) } \\
\text { and outbound (firing occurs as } \\
\text { rat leaves center) }\end{array}$ & $\begin{array}{l}\text { Grid cells have different modes } \\
\text { which account for upcoming } \\
\text { locations versus those recently } \\
\text { passed, serving both storage } \\
\text { and predictive functions } \\
\text { of hippocampus }\end{array}$ \\
\hline $\begin{array}{l}\text { xxiii. Caixeta, } \\
\text { Cornélio, } \\
\text { Scheffer-Teixeira, } \\
\text { Ribeiro, \& Tort } \\
\text { (2013) [65] }\end{array}$ & 8 male Wistar rats & iEEG & $\begin{array}{l}\text { Left } \\
\text { hippocampus }\end{array}$ & $\begin{array}{l}\text { - Open field } \\
\text { exploration } \\
\text { - Saline injection } \\
\text { - Ketamine injection }\end{array}$ & $\begin{array}{l}\text { - Ketamine increased motor } \\
\text { activity and gamma power } \\
\text { - Prominent phase-amplitude } \\
\text { coupling between theta- and } \\
\text { high-gamma }(60-100 \mathrm{~Hz}) \text { as well }\end{array}$ & $\begin{array}{l}\text { Some symptoms of } \\
\text { schizophrenia may be } \\
\text { explained by aberrant TGC } \\
\text { and/or theta-HFO coupling }\end{array}$ \\
\hline
\end{tabular}


Table 1 Animal findings regarding theta-gamma coupling (TGC) (Continued)

\begin{tabular}{|c|c|c|c|c|c|c|}
\hline & & & & & $\begin{array}{l}\text { Oscillations (HFO; } 110-160 \mathrm{~Hz}) \\
\text { pre-ketamine injection } \\
\text { - Theta-HFO coupling increased } \\
\text { with ketamine, while theta-gamma } \\
\text { coupling increased at the lowest } \\
\text { dosage but markedly disturbed } \\
\text { at the highest dosage }\end{array}$ & \\
\hline $\begin{array}{l}\text { xxiv. Newman, Gillet, } \\
\text { Climer, \& Hasselmo } \\
\text { (2013) [98] }\end{array}$ & 6 male Long-Evans rats & iEEG & $\begin{array}{l}\text { Entorhinal } \\
\text { cortex (medial) }\end{array}$ & $\begin{array}{l}\text { - Lap-running on } \\
\text { circular track } \\
\text { - With and without } \\
\text { scopolamine } \\
\text { injection }\end{array}$ & $\begin{array}{l}\text { - Robust phase-power TGC for } \\
\text { both low- }(20-40 \mathrm{~Hz}) \text { and high- } \\
(60-120 \mathrm{~Hz}) \text { gamma } \\
\text { - Scopolamine selectively } \\
\text { reduced high gamma power } \\
\text { at peak of theta }\end{array}$ & $\begin{array}{l}\text { - Encoding and retrieval may } \\
\text { occur at peak and through } \\
\text { of theta, respectively } \\
\text { - Acetylcholine influences } \\
\text { balance between encoding } \\
\text { and retrieval processes }\end{array}$ \\
\hline $\begin{array}{l}\text { xxv. Pernía-Andrade } \\
\text { \& Jonas (2014) [99] }\end{array}$ & Wistar rats & $\begin{array}{l}\text { Whole } \\
\text { cell } \\
\text { recording }\end{array}$ & $\begin{array}{l}\text { Hippocampal } \\
\text { (dentate gyrus) } \\
\text { granule cells }\end{array}$ & $\begin{array}{l}\text { - Anesthetized } \\
\text { - Free exploration }\end{array}$ & $\begin{array}{l}\text { - EPSCs coherent with LFP theta } \\
\text { oscillations; IPSCs coherent with } \\
\text { LFP gamma oscillations } \\
\text { - Action potentials phase locked to } \\
\text { theta-gamma oscillations in LFP }\end{array}$ & $\begin{array}{l}\text { - TGC in dentate gyrus may } \\
\text { reflect inhibitory gamma } \\
\text { currents phase locked to } \\
\text { theta currents and initiated } \\
\text { by excitation from } \\
\text { the entorhinal cortex } \\
\text { - Compound signal may serve } \\
\text { as temporal reference signal } \\
\text { for encoding in granule cells }\end{array}$ \\
\hline $\begin{array}{l}\text { xxvi. Yamamoto, } \\
\text { Suh, Takeuchi, } \\
\text { \& Tonegawa } \\
\text { (2014) [100] }\end{array}$ & $\begin{array}{l}\text { MECIII-TeTX MT Mice (allow } \\
\text { for reversible silencing of } \\
\text { synaptic transmission of } \\
\text { MEC layer III pyramidal } \\
\text { cells), control litter-mates }\end{array}$ & iEEG & $\begin{array}{l}\text { Hippocampus } \\
\text { (CA1), entorhinal } \\
\text { cortex (medial, } \\
\text { layer III) }\end{array}$ & $\begin{array}{l}\text { Open field } \\
\text { exploration }\end{array}$ & $\begin{array}{l}\text { - High-frequency and low-frequency } \\
\text { gamma modulate to different } \\
\text { phases of theta }\end{array}$ & $\begin{array}{l}\text { High-frequency gamma } \\
\text { contributes uniquely to } \\
\text { WM function }\end{array}$ \\
\hline \multicolumn{7}{|c|}{ In vitro or isolate studies } \\
\hline $\begin{array}{l}\text { xxvii. Cunningham, } \\
\text { Davies, Buhl, Kopell, } \\
\text { \& Whittington } \\
\text { (2003) }\end{array}$ & Male Sprague-Dawley rats & iEEG & $\begin{array}{l}\text { Hippocampus, } \\
\text { entorhinal cortex }\end{array}$ & In vitro & $\begin{array}{l}\text { - Amplitude of field gamma activity } \\
\text { modulated at theta frequency in } \\
\text { presence of kainate receptor activation }\end{array}$ & $\begin{array}{l}\text { EC can generate intrinsic } \\
\text { theta activity }\end{array}$ \\
\hline
\end{tabular}


Table 1 Animal findings regarding theta-gamma coupling (TGC) (Continued)

\begin{tabular}{|c|c|c|c|c|c|c|}
\hline $\begin{array}{l}\text { xxviii. Goutagny } \\
\text { et al. (2013) [102] }\end{array}$ & $\begin{array}{l}\text { TgCRND8 mice (develop at } \\
3 \text { months of age amyloid-beta } \\
\text { plaques typical of Alzheimer's } \\
\text { disease and accompanied by } \\
\text { similar cognitive decline) }\end{array}$ & iEEG & Hippocampus & $\begin{array}{l}\text { N/A (hippocampal } \\
\text { isolate) }\end{array}$ & $\begin{array}{l}\text { - Some TgCRND8 showed alterations } \\
\text { in phase-amplitude TGC prior to } \\
\text { accumulation of amyloid-beta } \\
\text { plaques or cognitive decline) } \\
\text { - Exclusive to theta coupling with } \\
\text { fast gamma, not slow gamma }\end{array}$ & $\begin{array}{l}\text { Declines in TGC are early } \\
\text { electrophysiological indicators } \\
\text { of hippocampal network } \\
\text { dysfunction }\end{array}$ \\
\hline $\begin{array}{l}\text { xxix. Pastoll, Solanka, } \\
\text { van Rossum, \& Nolan } \\
\text { (2013) [103] }\end{array}$ & $\begin{array}{l}\text { Adult Thy1-ChR2-YFP } \\
\text { line } 18 \text { mice }\end{array}$ & iEEG & $\begin{array}{l}\text { Entorhinal cortex } \\
\text { (medial) }\end{array}$ & $\begin{array}{l}\mathrm{N} / \mathrm{A} \text { (isolated brain } \\
\text { slices, though } \\
\text { with simulated } \\
\text { movement) }\end{array}$ & $\begin{array}{l}\text { - Optogenetic stimulation of medial } \\
\text { entorhinal cortex at theta frequency } \\
\text { sufficient to produce } \\
\text { nested gamma activity with both } \\
\text { phase and amplitude coupled } \\
\text { to theta phase } \\
\text { - Nested gamma is mediated by } \\
\text { feedback inhibition }\end{array}$ & $\begin{array}{l}\text { Local medial entorhinal cortex } \\
\text { circuit produces TGC with } \\
\text { "clock-like" (p. 153) consistency; } \\
\text { coupled signals may serve as } \\
\text { temporal references for other } \\
\text { neuronal computations }\end{array}$ \\
\hline \multicolumn{7}{|l|}{ Primate studies } \\
\hline $\begin{array}{l}\text { xxx. Lakatos et al. } \\
\text { (2005) [104] }\end{array}$ & 4 male macaque monkeys & iEEG & $\begin{array}{l}\text { Primary auditory } \\
\text { cortex }\end{array}$ & $\begin{array}{l}\text { Passive listening } \\
\text { task }\end{array}$ & $\begin{array}{l}\text { Spontaneous gamma-activity found } \\
\text { to fluctuate at theta-frequency, and } \\
\text { theta-activity found to subsequently } \\
\text { fluctuate at delta frequency }\end{array}$ & $\begin{array}{l}\text { Oscillatory activity is organized } \\
\text { in a hierarchical manner, not } \\
\text { exclusively limited to gamma } \\
\text { and theta activity }\end{array}$ \\
\hline $\begin{array}{l}\text { xxxi. Voloh, Valiante, } \\
\text { Everling, \& Womelsdorf } \\
\text { (2015) [105] }\end{array}$ & 2 macaque monkeys & iEEG & $\begin{array}{l}\text { Medial and lateral } \\
\text { PFC (anterior } \\
\text { cingulate cortex) }\end{array}$ & Attention task & $\begin{array}{l}\text { - Theta-phase to gamma-amplitude } \\
\text { TGC between various sites in ACC } \\
\text { and PFC during task performance, } \\
\text { but not before errors }\end{array}$ & $\begin{array}{l}\text { TGC essential to integration } \\
\text { of varied and distributed } \\
\text { activities, including attention, } \\
\text { in neural networks }\end{array}$ \\
\hline
\end{tabular}

WM working memory, TGC theta-gamma coupling, iEEG intracranial EEG, LFP local field potential, CSD current source density, EC entorhinal cortex, HFO high-frequency oscillation, ACC anterior cingulate cortex, PFC prefrontal cortex 
Table 2 Human findings regarding theta-gamma coupling (TGC)

\begin{tabular}{|c|c|c|c|c|c|c|c|}
\hline $\begin{array}{l}\text { Authors \& Year } \\
\text { of Publication }\end{array}$ & Subjects & Measure & $\begin{array}{l}\text { Region of Brain/ } \\
\text { Scalp Examined }\end{array}$ & $\begin{array}{l}\text { Task Performed/Behavior } \\
\text { during Measurement }\end{array}$ & $\begin{array}{l}\text { WM Subprocess(es) } \\
\text { Analyzed }\end{array}$ & Relevant Findings & Conclusions \\
\hline \multicolumn{8}{|c|}{ Studies utilizing intracranial EEG (iEEG) } \\
\hline $\begin{array}{l}\text { i. Fell et al. } \\
\text { (2003) [106] }\end{array}$ & $\begin{array}{l}9 \text { patients with } \\
\text { pharmacoresistant } \\
\text { temporal lobe epilepsy }\end{array}$ & iEEG & $\begin{array}{l}\text { Hippocampus, } \\
\text { entorhinal cortex }\end{array}$ & $\begin{array}{l}\text { Single-trial word list } \\
\text { learning paradigm }\end{array}$ & Encoding & $\begin{array}{l}\text { - Phase-phase TGC across the } \\
\text { rhinal cortex and hippocampus } \\
\text { during WM encoding } \\
\text { - Coupling greater for words } \\
\text { successfully recalled }\end{array}$ & $\begin{array}{l}\text { TGC crucial to actual memory } \\
\text { formation rather than general } \\
\text { state of WM encoding }\end{array}$ \\
\hline $\begin{array}{l}\text { ii. Mormann } \\
\text { et al. (2005) [76] }\end{array}$ & $\begin{array}{l}12 \text { patients with } \\
\text { pharmacoreistant } \\
\text { temporal lobe epilepsy } \\
(5 \text { women, aged } \\
40.3 \pm 10.1 \text { years })\end{array}$ & iEEG & $\begin{array}{l}\text { Hippocampus, } \\
\text { rhinal cortex }\end{array}$ & $\begin{array}{l}\text { Continuous visual word } \\
\text { recognition paradigm }\end{array}$ & $\begin{array}{l}\text { Continuous } \\
\text { recognition } \\
\text { (Combination of } \\
\text { encoding and } \\
\text { retrieval) }\end{array}$ & $\begin{array}{l}\text { - Modulation of gamma- } \\
\text { amplitude by theta- phase } \\
\text { in both rhinal cortex } \\
\text { hippocampus } \\
\text { - Rhinal modulation greatest } \\
\text { for correct rejections, } \\
\text { hippocampal modulation } \\
\text { greatest for hits }\end{array}$ & $\begin{array}{l}\text { Phase-amplitude TGC may } \\
\text { underlie encoding processes } \\
\text { in rhinal cortex and retrieval } \\
\text { processes in hippocampus }\end{array}$ \\
\hline $\begin{array}{l}\text { iii. Axmacher } \\
\text { et al. (2010) [33] }\end{array}$ & $\begin{array}{l}14 \text { participants with } \\
\text { pharmacoresistant } \\
\text { temporal lobe epilepsy } \\
\text { ( } 3 \text { female, aged } \\
38.3 \pm 11.8 \text { years) }\end{array}$ & iEEG & Hippocampus & $\begin{array}{l}\text { Visual analogue of } \\
\text { Sternberg task }\end{array}$ & Maintenance & $\begin{array}{l}\text { - Theta phase to gamma } \\
\text { power TGC increased during } \\
\text { maintenance compared } \\
\text { to baseline } \\
\text { - More precise coupling } \\
\text { associated with faster } \\
\text { reaction times } \\
\text { - Increased WM load related } \\
\text { to less modulation of gamma }\end{array}$ & $\begin{array}{l}\text { - Medial temporal lobe } \\
\text { involved in WM when } \\
\text { multiple stimuli are to be } \\
\text { maintained } \\
\text { - TGC functionally relevant } \\
\text { to WM processes }\end{array}$ \\
\hline $\begin{array}{l}\text { iv. Canolty et al. } \\
\text { (2006) [61] }\end{array}$ & $\begin{array}{l}5 \text { females (aged } \\
24 \text { to } 45 \text { years) }\end{array}$ & iEEG & $\begin{array}{l}\text { Frontal, temporal, } \\
\text { parietal lobes }\end{array}$ & $\begin{array}{l}\text { - Passive listening } \\
\text { - Active listening/ } \\
\text { target detection } \\
\text { - Picture naming } \\
\text { - Verb generation } \\
\text { - Finger tapping } \\
\text { - Mouth movement } \\
\text { \& articulation } \\
\text { - Auditory/vibrotactile } \\
\text { stimulus presentation }\end{array}$ & Unclear & $\begin{array}{l}\text { - Strong modulation of } \\
\text { gamma throughout examined } \\
\text { areas by theta phase across } \\
\text { all tasks; highest modulation } \\
\text { in high gamma }(80-150 \mathrm{~Hz}) \\
\text { - Largest gamma amplitudes } \\
\text { occur at trough of theta }\end{array}$ & $\begin{array}{l}\text { TGC coordinates various } \\
\text { cortical regions for } \\
\text { purposeful activity }\end{array}$ \\
\hline
\end{tabular}


Table 2 Human findings regarding theta-gamma coupling (TGC) (Continued)

\begin{tabular}{|c|c|c|c|c|c|c|c|}
\hline & & & & $\begin{array}{l}\text { with unimodal target } \\
\text { detection } \\
\text { - Auditory N-back task } \\
\text { - Visual search task } \\
\text { - Visual silent reading task } \\
\text { - Facial emotion recognition } \\
\text { task } \\
\text { - Motor, auditory and tactile } \\
\text { imagery }\end{array}$ & & & \\
\hline $\begin{array}{l}\text { v. van der Meij, } \\
\text { Kahana, \& Maris } \\
\text { (2012) [107] }\end{array}$ & $\begin{array}{l}27 \text { patients ( } 12 \text { female) } \\
\text { with pharmacoresistant } \\
\text { epilepsy }\end{array}$ & iEEG & $\begin{array}{l}\text { Frontal, temporal, } \\
\text { parietal, occipital, } \\
\text { limbic }\end{array}$ & Sternberg task & $\begin{array}{l}\text { Encoding, } \\
\text { maintenance }\end{array}$ & $\begin{array}{l}\text { Widespread phase-amplitude } \\
\text { coupling between multiple } \\
\text { frequency bands across } \\
\text { distances greater than } 10 \mathrm{~cm} \\
\text { between sensors spread } \\
\text { throughout cortex and other } \\
\text { measured areas }\end{array}$ & $\begin{array}{l}\text { - Phase-amplitude coupling is } \\
\text { pervasive, has a wide spatial } \\
\text { distribution, and varies greatly } \\
\text { in terms of frequencies } \\
\text { involved and phases preferred } \\
\text { - As such, phase-amplitude } \\
\text { coupling likely involved in } \\
\text { various forms of selective } \\
\text { communication between } \\
\text { brain areas }\end{array}$ \\
\hline $\begin{array}{l}\text { vi. Maris, van } \\
\text { Vugt, \& Kahana } \\
\text { (2011) [108] }\end{array}$ & $\begin{array}{l}26 \text { patients with } \\
\text { implanted electrodes } \\
\text { for purpose of } \\
\text { presurgical diagnosis }\end{array}$ & iEEG & $\begin{array}{l}\text { Varied from patient- } \\
\text { to-patient, but } \\
\text { included frontal, } \\
\text { temporal and } \\
\text { parietal sites }\end{array}$ & Sternberg task & $\begin{array}{l}\text { Encoding, } \\
\text { maintenance }\end{array}$ & $\begin{array}{l}\text { - Phase to amplitude coupling } \\
\text { widely distributed across } \\
\text { frequency as well as location; } \\
\text { included frontal, temporal } \\
\text { and parietal sites } \\
\text {-TGC most prominent in } \\
\text { within-electrode analyses } \\
\text { - Phase-amplitude coupling } \\
\text { increased in some areas } \\
\text { during WM and decreased } \\
\text { in others } \\
\text { - In most coupling patterns, } \\
\text { high-frequency bursts are } \\
\text { synchronized across locations }\end{array}$ & $\begin{array}{l}\text { - WM processes affect coupling } \\
\text { heterogeneously, depending } \\
\text { on location and frequencies } \\
\text { involved } \\
\text { - Phase-amplitude coupling } \\
\text { important to coordination of } \\
\text { neural activity spatiotemporally }\end{array}$ \\
\hline
\end{tabular}


Table 2 Human findings regarding theta-gamma coupling (TGC) (Continued)

\begin{tabular}{|c|c|c|c|}
\hline $\begin{array}{l}\text { vii. Chaieb et al. } \\
\text { (2015) [34] }\end{array}$ & $\begin{array}{l}14 \text { patients with } \\
\text { pharmacoresistant } \\
\text { temporal lobe epilepsy }\end{array}$ & iEEG & Hippocampus \\
\hline
\end{tabular}

Studies utilizing scalp-level recordings

Petsche, Geissler, 10 right-handed

\& Möller (2002) [70] (aged 25 to

35 years)

ix. Baerbel Schack 23 females (aged

$\&$ Weiss (2005) [109] $23.7 \pm 2.7$ years)

$\begin{array}{ll}\begin{array}{l}\text { x. Sauseng, } \\ \text { Klimesch, Gruber, }\end{array} & \begin{array}{l}22 \text { analyzed patients } \\ \text { (19 females, average } \\ \text { (2008) [69] }\end{array} \\ \begin{array}{ll}\text { age of 23.6 years) } \\ \text { xi. Sauseng et al. }\end{array} & \begin{array}{l}21 \text { right-handed } \\ \text { (2009) [32] }\end{array} \\ & \begin{array}{l}\text { participants (15 } \\ \text { females, aged } \\ 23.9 \pm 4.4 \text { years) }\end{array}\end{array}$

\section{Scalp}

EEG

frontopol, prefrontal,

Scalp Scalp-wide

EEG

Scalp

\section{Scalp Scalp-wide}

Sternberg tasks featuring

random figures

facial stimuli

Single-trial word list

learning paradigm

Cued visual attention task

Visuospatial delayed match-to-sample task
Maintenance

- Increases in phase-phase TGC

observed for multi-item but not

single-item trials for coupling

factor of 6 (e.g., 6 gamma

cycles to 1 theta cycle)

- Changes in coupling

measures across WM loads

predicted WM capacities

\section{Encoding,}

maintenance and

- Phase-coupling between

theta activity over frontal areas

and gamma activity over

frontopolar areas during

maintenance of number words

Encoding

- Increased frontocentral

phase-phase TGC for words

later recalled vs. those not

- Phase-phase TGC also

observed between Fz and $\mathrm{Cz}$

Target presentation - Phase-phase TGC over

posterior areas enhanced by posterior areas enhanced by areas reflects matching of

- Stronger TGC over areas

contralateral to cued visual field

Maintenance

Phase-amplitude TGC over

bilateral parietal and

occipital sites

- Phase-phase TGC modulated

by WM load over right

posterior sites, exclusive to

relevant information

Degree of phase-phase TGC

modulation by WM load

predictive of WM capacity
TGC is prominent during

man maintenance of

formation in WM,

supporting Lisman \& Idiart's

1] model

Gabor expansion is a useful

means of assessing phase-phase

TGC from scalp EEG

Phase-phase TGC over posterior visual input

\section{TGC, and particularly}

phase-phase relationships, are central to visual WM function - Independent mechanisms for maintenance of relevant vs. suppression of irrelevant info 
Table 2 Human findings regarding theta-gamma coupling (TGC) (Continued)

\begin{tabular}{|c|c|c|c|c|c|c|c|}
\hline $\begin{array}{l}\text { xii. (Holz, Glennon, } \\
\text { Prendergast, \& } \\
\text { Sauseng (2010) [68] }\end{array}$ & $\begin{array}{l}18 \text { participants } \\
\text { (13 female, aged } \\
23.67 \pm 2.99 \text { years) }\end{array}$ & $\begin{array}{l}\text { Scalp } \\
\text { EEG }\end{array}$ & Scalp-wide & $\begin{array}{l}\text { Visuospatial delayed } \\
\text { match-to-sample task }\end{array}$ & Retrieval & $\begin{array}{l}\text { - Increased phase-phase TGC } \\
\text { over parieto-occipital brain } \\
\text { regions soon after } \\
\text { probe presentation } \\
\text { - Matching increased TGC } \\
\text { in right hemisphere; } \\
\text { non-matching increased TGC } \\
\text { in left hemisphere }\end{array}$ & $\begin{array}{l}\text { Comparison of stored } \\
\text { information to visual } \\
\text { inputs leads to phase-phase } \\
\text { TGC over parieto-occipital } \\
\text { regions }\end{array}$ \\
\hline $\begin{array}{l}\text { xiii. Park, Lee, } \\
\text { \& Lee (2011) [36] }\end{array}$ & $\begin{array}{l}31 \text { older participants } \\
\text { (19 females, aged } \\
66.45 \pm 5.75 \text { years })\end{array}$ & $\begin{array}{l}\text { Scalp } \\
\text { EEG }\end{array}$ & Scalp-wide & $\begin{array}{l}\text { Spatial delayed match- } \\
\text { to-sample task }\end{array}$ & Maintenance & $\begin{array}{l}\text { - TGC between theta phase } \\
\text { and gamma power across } \\
\text { scalp, enhanced in left } \\
\text { centro-parietal regions } \\
\text { - Strength of TGC at FP1 } \\
\text { predicted task performance } \\
\text { - Strength of TGC at P8 } \\
\text { predicted delayed figure } \\
\text { recall performance }\end{array}$ & $\begin{array}{l}\text { TGC is important to visual } \\
\text { memory function (e.g., } \\
\text { parietal correlation) but } \\
\text { may also reflect attentional } \\
\text { processes (e.g., prefrontal } \\
\text { correlation) }\end{array}$ \\
\hline $\begin{array}{l}\text { xiv. Park, Jhung, } \\
\text { Lee, \& An } \\
\text { (2013) [35] }\end{array}$ & $\begin{array}{l}13 \text { right-handed } \\
\text { participants ( } 5 \\
\text { females, aged } \\
20.8 \pm 3.1 \text { years) }\end{array}$ & $\begin{array}{l}\text { Scalp } \\
\text { EEG }\end{array}$ & Scalp-wide & $\begin{array}{l}\text { Visuospatial 2-back } \\
\text { task, vigilance task }\end{array}$ & Maintenance & $\begin{array}{l}\text { TGC between theta phase } \\
\text { and gamma power most } \\
\text { prominent over prefrontal } \\
\text { areas in WM task but not } \\
\text { vigilance task }\end{array}$ & $\begin{array}{l}\text { TGC recorded from scalp } \\
\text { represents WM processes } \\
\text { rather than attentional } \\
\text { processes }\end{array}$ \\
\hline $\begin{array}{l}\text { XV. Lee \& Yang } \\
\text { (2014) [110] }\end{array}$ & $\begin{array}{l}9 \text { right-handed } \\
\text { males (aged } \\
21.22 \pm 0.97 \text { years) }\end{array}$ & $\begin{array}{l}\text { Scalp } \\
\text { EEG }\end{array}$ & Scalp-wide & $\begin{array}{l}\text { Visuospatial delayed } \\
\text { match-to-sample task }\end{array}$ & Maintenance & $\begin{array}{l}\text { Strength of phase-phase } \\
\text { TGC at P8 predicted } \\
\text { task performance }\end{array}$ & $\begin{array}{l}\text { Phase-phase TGC can be } \\
\text { used as a measure of } \\
\text { WM ability }\end{array}$ \\
\hline $\begin{array}{l}\text { xvi. Vosskuhl, } \\
\text { Huster, \& Herrmann } \\
\text { (2015) [111] }\end{array}$ & $\begin{array}{l}33 \text { right-handed } \\
\text { participants }(14 \\
\text { females, aged } \\
25.74 \pm 2.69 \text { years })\end{array}$ & $\begin{array}{l}\text { Scalp } \\
\text { EEG }\end{array}$ & $\begin{array}{l}\mathrm{F} 3, \mathrm{Fz}, \mathrm{F} 4, \mathrm{C} 3 \\
\mathrm{Cz}, \mathrm{C} 4\end{array}$ & $\begin{array}{l}\text { Transcranial alternating } \\
\text { current stimulation; digit } \\
\text { span task; 3-back task }\end{array}$ & $\begin{array}{l}\text { Encoding during } \\
\text { stimulation }\end{array}$ & $\begin{array}{l}\text { - Short-term memory capacity } \\
\text { as assessed using forward } \\
\text { digit span was increased } \\
\text { during stimulation } \\
\text { - Theta amplitude increased } \\
\text { after stimulation }\end{array}$ & $\begin{array}{l}\text { Supports theory that the } \\
\text { length of theta cycle (e.g., } \\
\text { theta frequency) limits how } \\
\text { many gamma cycles can be } \\
\text { superimposed upon it }\end{array}$ \\
\hline
\end{tabular}


Table 2 Human findings regarding theta-gamma coupling (TGC) (Continued)

\begin{tabular}{|c|c|c|c|c|c|c|c|}
\hline $\begin{array}{l}\text { xvii. Heusser, } \\
\text { Poeppel, Ezzyat, } \\
\text { \& Davachi } \\
\text { (2016) [77] }\end{array}$ & $\begin{array}{l}20 \text { right-handed } \\
\text { males (aged } \\
\text { 21-25; mean } \\
\text { age 28) }\end{array}$ & MEG & $\begin{array}{l}\text { Scalp-wide; left } \\
\text { lateral and posterior } \\
\text { clusters of sensors } \\
\text { used for analyses }\end{array}$ & $\begin{array}{l}\text { Visual temporal order } \\
\text { memory paradigm }\end{array}$ & Encoding & $\begin{array}{l}\text { - Peak of gamma power shifted } \\
\text { progressively later with memory } \\
\text { stimuli progressively later in } \\
\text { presented sequence } \\
\text { - Relative gamma peaks } \\
\text { reflected the order of stimulus } \\
\text { presentation in sequence }\end{array}$ & $\begin{array}{l}\text { Strongly supports notion } \\
\text { that the sequential order } \\
\text { of stimuli is encoded using } \\
\text { a theta-gamma code }\end{array}$ \\
\hline $\begin{array}{l}\text { xviii. Alekseichuk, } \\
\text { Turi, de Lara, } \\
\text { Antal, \& Paulus } \\
\text { (2016) [112] }\end{array}$ & $\begin{array}{l}47 \text { healthy } \\
\text { people ( } 25 \\
\text { females, aged } \\
\text { 19-28) }\end{array}$ & $\begin{array}{l}\text { Scalp } \\
\text { EEG }\end{array}$ & Scalp-wide & $\begin{array}{l}\text { Transcranial alternating } \\
\text { current stimulation; } \\
\text { 2-back visuospatial } \\
\text { memory test }\end{array}$ & $\begin{array}{l}\text { Encoding/retrieval } \\
\text { during stimulation }\end{array}$ & $\begin{array}{l}\text { - Transcranial alternating current } \\
\text { stimulation improved task } \\
\text { performance and increased } \\
\text { phase connectivity across scalp } \\
\text { - } 80 \mathrm{~Hz} \text { to } 100 \mathrm{~Hz} \text { stimulation } \\
\text { during theta peak produced } \\
\text { optimal effects }\end{array}$ & $\begin{array}{l}\text { - Transcranial current in } \\
\text { gamma frequency range } \\
\text { improved WM performance } \\
\text { - High gamma may be } \\
\text { particularly important to } \\
\text { WM-related coupling }\end{array}$ \\
\hline
\end{tabular}

WM working memory, TGC theta-gamma coupling, iEEG intracranial EEG, LFP local field potential, CSD current source density, EC entorhinal cortex, HFO high-frequency oscillation, ACC anterior cingulate cortex, PFC prefrontal cortex 
cycles (xvi), while another used MEG to show progressively later peaks in gamma activity relative to concurrent theta activity for progressively later memory items in an encoding sequence (xvii). In addition, measures of theta-gamma coupling have similarly been related to WM performance in experimentation with humans (e.g., vii, xi, xiii, xv). Thus, theta-gamma coupling has been repeatedly associated with WM processes in both the animal and human literature.

\section{The role of abnormal theta-/gamma- oscillations in schizophrenia}

Given the well-documented importance of theta-gamma modulations to memory processes in animal and human studies alike, abnormal interactions between theta- and gamma-oscillations have been proposed to explain the prominent WM deficits observed in PSZ [37, 38]. In particular, predictive relationships demonstrated between indices of theta-gamma coupling and WM capacity [32,34] as well as performance on WM tasks [36, 107] in healthy people suggest that impaired WM performance observed in PSZ may be attributed to deficient theta-gamma coupling. Formal investigation of this proposal has been scarce to date. As such, in addition to the few direct examinations of theta-gamma coupling in PSZ, demonstrated abnormalities in thetaand gamma- oscillations independently are likewise reported, and their potential relevance to interactions between the two frequency bands is discussed.

\section{Theta-gamma coupling in people with schizophrenia}

Few studies have directly examined interactions between theta- and gamma- activity in PSZ. Allen and colleagues [62] examined phase-amplitude coupling between low- and highfrequencies using independent component analysis (ICA) of EEG recorded from PSZ during performance of an auditory oddball task. In this ICA, data for comodulation between various frequencies were decomposed into separate components based on spectral and spatial composition. One such component showing widespread coupling between lower-frequency phases and higher-frequency amplitudes across the entire scalp was less prominent in PSZ data compared to that of healthy controls, and another component depicting modulation of high frequency amplitude by low frequency phase at frontotemporal sites was more prominent for PSZ than controls. Another component revealed strong theta-gamma coupling over occipital-parietal areas, but the loading parameters for said component did not differ between groups (e.g., the component was equivalently strong in PSZ and healthy controls). First-degree relatives of people with the disorder were included in this study and showed coupling intermediate to that of PSZ and healthy controls, though their data were not tested statistically due to power limitations. The authors interpret their results to indicate that abnormal cross-frequency coupling may represent an endophenotype for schizophrenia, particularly in light of genetic associations they report for genotypes for certain genes and loading parameters for various independent components in PSZ. However, though patterns of cross-frequency coupling were altered in PSZ, theta-gamma coupling is still prominent, especially over posterior sites. As such, theta-gamma coupling may not be significantly altered in the disorder, at least in the context of novelty detection. No correlations between measures of cross-frequency coupling and performance were observed [62]. Thus, this study provides evidence for preserved theta-gamma phase-amplitude coupling in PSZ; however, given the minimal role WM plays in the oddball paradigm, the theta-gamma interactions examined in this study may be distinct from those related to WM. 
The only other published work examining theta-gamma cross-frequency interactions in PSZ comes from Kirihara and colleagues [63]. Using scalp EEG recorded during presentation of $40-\mathrm{hz}$ steady state auditory stimuli, the authors found reduced intertrial phase coherence, increased theta amplitude, and undisturbed theta-gamma phaseamplitude coupling in PSZ relative to healthy controls. The authors interpret these findings to indicate a preserved hierarchical organization of theta and gamma activity in PSZ despite abnormalities in theta- and gamma- activity independently [63]. Notably, the simple auditory processing task used in this study does not tap memory processes, for which theta-gamma interactions have been shown to essential and greatly impaired in PSZ; thus, the reported intact theta-gamma coupling should not be interpreted as indicative of preserved coupling at large.

Two additional studies investigated theta-gamma coupling under influence of ketamine, an NMDAR antagonist which is used as a pharmacological model of schizophrenia in both animals and humans. Neymotin and colleagues [64] used a computer model of hippocampal CA3 to investigate the effect of the application of simulated ketamine. Ketamine administration disrupted theta-gamma coupling in the simulation, apparently due to its antagonistic effect on NMDA receptors on orienslacunosum moleculare cells (OLM); normal coupling was restored via injection of a continuous, depolarizing current into these OLM cells, suggesting a potential intervention for treating deficits in WM and other cognitive processes in PSZ. Similarly, Caixeta and colleagues [65] found that ketamine altered theta-gamma coupling in the hippocampus of rats, again suggesting the importance of theta-gamma interactions to cognitive deficits in PSZ. Still, theta-gamma coupling in the context of memory function in PSZ has not yet been directly examined.

\section{Theta- and gamma- band abnormalities in people with schizophrenia}

Though research on theta-gamma coupling in schizophrenia is sparse, findings from the individual bands support the notion of WM impairments in PSZ stemming from aberrant theta-gamma interactions. Abnormalities in theta- and gamma-activity during WM reported in PSZ are summarized in Table 3. These abnormalities include deficits in theta-synchrony (e.g., Table 3, reference iv) and aberrations in overall thetaamplitudes (e.g., i-iv) as well as modulations in response to task demands (e.g., i, iv), though results are variable regarding whether PSZ show enhanced or reduced amplitudes/modulation as compared with controls. Despite this variability, all studies show a failure of PSZ to modulate theta amplitudes with changes in task demands compared to controls, suggesting an inefficient allocation of cognitive resources (suggested by i, iii, iv) and/or deficiencies in neural responses associated with sequential presentation of stimuli (suggested by ii). Amplitudes of gamma-activity during WM have similarly been found to be abnormal in PSZ (e.g., v-ix), though findings are again mixed as to whether these abnormalities are enhancements or reductions compared to controls; as with theta, however, PSZ frequently fail to modulate (e.g., vi, viii-ix) or abnormally modulate (vii) gamma responses with changes in task demands. Again, these findings suggest that PSZ either lack or misallocate cognitive resources as compared to healthy controls. Discrepancies in whether oscillatory responses are increased or decreased in PSZ relative to controls have many potential sources, including heterogeneity in symptomatology, differences in stage of illness (e.g., early onset 
Table 3 Theta and gamma abnormalities in people with schizophrenia (PSZ) during working memory processes

\begin{tabular}{|c|c|c|c|c|c|c|c|}
\hline $\begin{array}{l}\text { Authors \& Year } \\
\text { of Publication }\end{array}$ & Subjects & Measure & $\begin{array}{l}\text { Region of } \\
\text { Brain/Scalp } \\
\text { Examined }\end{array}$ & $\begin{array}{l}\text { Task Performed/ } \\
\text { Behavior during } \\
\text { Measurement }\end{array}$ & $\begin{array}{l}\text { WM Subprocess(es) } \\
\text { Analyzed }\end{array}$ & Relevant Findings & Conclusions \\
\hline \multicolumn{8}{|l|}{ Theta abnormalities } \\
\hline $\begin{array}{l}\text { i. Schmiedt, Brand, } \\
\text { Hildebrandt, \& } \\
\text { Başar-Eroğlu } \\
\text { (2005) [113] }\end{array}$ & $\begin{array}{l}-10 \text { inpatient PSZ } \\
\text { (aged } 34 \pm 6 \text { years) } \\
-10 \text { healthy controls } \\
\text { (aged } 24 \pm 4 \text { years) }\end{array}$ & Scalp EEG & $\begin{array}{l}\mathrm{Fz}, \mathrm{Cz}, \mathrm{Pz}, \mathrm{Oz}, \\
\mathrm{F} 3, \mathrm{~F} 4, \mathrm{T5}, \mathrm{T6}\end{array}$ & $\begin{array}{l}\text { Visual variable } \\
\mathrm{N} \text {-back with action } \\
\text { monitoring/ } \\
\text { rule-switching }\end{array}$ & $\begin{array}{l}\text { Maintenance, } \\
\text { encoding }\end{array}$ & $\begin{array}{l}\text { - Widespread reduced } \\
\text { theta-amplitude in PSZ } \\
\text { during maintenance compared } \\
\text { to controls } \\
\text { - Enhanced amplitude in PSZ at left } \\
\text { temporal locations in first } 250 \text { ms } \\
\text { post-stimulus } \\
\text { - PSZ failed to modulate frontal } \\
\text { theta amplitude with task changes, } \\
\text { whereas controls' amplitudes } \\
\text { increased with task difficulty }\end{array}$ & $\begin{array}{l}\text { - Increased temporal theta indicates } \\
\text { pathological processes in frontal } \\
\text { and temporal regions in PSZ } \\
\text { - Failure to modulate theta suggests } \\
\text { deficits in executive processes } \\
\text { related to WM }\end{array}$ \\
\hline $\begin{array}{l}\text { ii. Haenschel } \\
\text { et al. (2009) [29] }\end{array}$ & $\begin{array}{l}\text { - } 14 \text { patients with early } \\
\text { onset schizophrenia } \\
\text { (onset prior to age } \\
18 ; 5 \text { females, aged } \\
17.76 \pm 1.44 \text { years) } \\
-14 \text { controls (aged } \\
17.37 \pm 1.41 \text { years) }\end{array}$ & Scalp EEG & Scalp-wide & $\begin{array}{l}\text { Delayed visual } \\
\text { discrimination task }\end{array}$ & $\begin{array}{l}\text { Encoding, } \\
\text { maintenance } \\
\text { and retrieval }\end{array}$ & $\begin{array}{l}\text { - PSZ showed reduced } \\
\text { theta-amplitudes during } \\
\text { encoding and retrieval as } \\
\text { compared to controls } \\
\text { - Evoked posterior theta } \\
\text { decreased with increasing } \\
\text { WM load during encoding } \\
\text { in controls but not PSZ } \\
\text { - Evoked anterior theta during } \\
\text { encoding predictive of } \\
\text { performance in controls but } \\
\text { not PSZ }\end{array}$ & $\begin{array}{l}\text { - Reduced evoked theta during } \\
\text { encoding may reflect impaired } \\
\text { phase resetting to stimuli; } \\
\text { reductions during retrieval } \\
\text { may reflect a failure in PSZ } \\
\text { to recognize previously } \\
\text { encountered stimuli } \\
\text { - Lack of decrease in theta } \\
\text { amplitude in PSZ may reflect } \\
\text { deficient responses to initial } \\
\text { stimuli as well as habituation } \\
\text { processes }\end{array}$ \\
\hline $\begin{array}{l}\text { iii. Missonnier } \\
\text { et al. (2012) [114] }\end{array}$ & $\begin{array}{l}\text { - } 15 \text { patients with } \\
\text { first-episode psychosis } \\
\text { ( } 3 \text { females, aged } 21.9 \pm \\
2.6 \text { years, } 6 \text { diagnosed } \\
\text { schizophreniform, } \\
9 \text { schizophrenia) } \\
\text { - } 18 \text { controls ( } 9 \text { females, } \\
\text { aged } 24.4 \pm 1.5 \text { years) }\end{array}$ & Scalp EEG & Scalp-wide & $\begin{array}{l}\text { - Visual oddball task } \\
\text { - Visual N-back task } \\
\text { - Simple detection task }\end{array}$ & $\begin{array}{l}\text { Maintenance, } \\
\text { encoding }\end{array}$ & $\begin{array}{l}\text { - PSZ showed increased sustained } \\
\text { theta amplitudes in frontal areas } \\
\text { for the retention period of } \\
\text { detection and 1-back tasks, as } \\
\text { well as delayed maximums of } \\
\text { phasic event-related theta } \\
\text { synchronization, compared } \\
\text { to controls }\end{array}$ & $\begin{array}{l}\text { PSZ fail to appropriately } \\
\text { modulate brain activation } \\
\text { to address increasing } \\
\text { demands on WM }\end{array}$ \\
\hline
\end{tabular}


Table 3 Theta and gamma abnormalities in people with schizophrenia (PSZ) during working memory processes (Continued)

\begin{tabular}{|c|c|c|c|c|c|c|c|}
\hline $\begin{array}{l}\text { iv. Griesmayr } \\
\text { et al. (2014) [115] }\end{array}$ & $\begin{array}{l}\text { - } 21 \text { PSZ }(5 \text { females, } \\
\text { aged } 22 \text { to } 46.02 \\
\text { years, mean } \\
\text { age }=31.96 \text { years) } \\
\text { - } 21 \text { healthy controls } \\
\text { (5 females, aged } 20.05 \\
\text { to } 47.02 \text { years, mean } \\
\text { age }=31.55 \text { ) }\end{array}$ & Scalp EEG & Scalp-wide & $\begin{array}{l}\text { Visuospatial delayed } \\
\text { match-to-sample } \\
\text { task with manipulation }\end{array}$ & $\begin{array}{l}\text { Maintenance/ } \\
\text { manipulation }\end{array}$ & $\begin{array}{l}\text { - Reduced phase-synchrony } \\
\text { in PSZ: primarily reductions } \\
\text { between frontal and posterior } \\
\text { regions, but reductions within } \\
\text { posterior areas also observed } \\
\text { during high WM-load } \\
\text { - PSZ showed increased theta } \\
\text { amplitude for manipulation } \\
\text { versus maintenance processes; } \\
\text { controls showed no such difference } \\
\text { - Controls demonstrated increase } \\
\text { in posterior theta amplitude with } \\
\text { increasing load; PSZ did not }\end{array}$ & $\begin{array}{l}\text { - Deficits in theta phase-synchrony } \\
\text { represent binding difficulties } \\
\text { in PSZ } \\
\text { - Modulation of theta amplitude in } \\
\text { PSZ may suggest inefficient } \\
\text { allocation of cognitive resources } \\
\text { rather than pure WM processes }\end{array}$ \\
\hline \multicolumn{8}{|c|}{ Gamma abnormalities } \\
\hline $\begin{array}{l}\text { v. Kissler, Müller, } \\
\text { Fehr, Rockstroh, } \\
\text { \& Elbert } \\
\text { (2000) [116] }\end{array}$ & $\begin{array}{l}\text { - } 15 \text { PSZ ( } 4 \text { females, } \\
\text { aged } 30.2 \pm 6.5 \text { years) } \\
\text { - } 15 \text { healthy controls } \\
\text { (4 females, aged } \\
35.8 \pm 9.4 \text { years) }\end{array}$ & MEG & Whole-head & Mental arithmetic task & Manipulation & $\begin{array}{l}\text { Controls showed increases in } \\
\text { frontotemporal gamma activity } \\
\text { during arithmetic; PSZ did not, } \\
\text { but instead showed reduced } \\
\text { gamma-amplitudes at temporal } \\
\text { and occipital areas }\end{array}$ & $\begin{array}{l}\text { - Gamma-activity can be } \\
\text { generated without } \\
\text { external stimuli } \\
\text { - Schizophrenia may be } \\
\text { associated with abnormalities } \\
\text { in thalamocortical loop }\end{array}$ \\
\hline $\begin{array}{l}\text { vi. Başar-Eroğlu } \\
\text { et al. (2007) [117] }\end{array}$ & $\begin{array}{l}-10 \text { PSZ (aged } \\
34 \pm 6 \text { years) } \\
\text { - } 10 \text { healthy controls } \\
\text { (aged } 24 \pm 4 \text { years) }\end{array}$ & Scalp EEG & $\begin{array}{l}\mathrm{Fz}, \mathrm{Cz}, \mathrm{Pz}, \mathrm{Oz}, \\
\text { F3, F4, T5, T6 }\end{array}$ & $\begin{array}{l}\text { Visual variable N-back } \\
\text { with action monitoring/ } \\
\text { rule-switching }\end{array}$ & $\begin{array}{l}\text { Maintenance, } \\
\text { encoding/retrieval }\end{array}$ & $\begin{array}{l}\text { - Trend towards increased } \\
\text { gamma-power across scalp } \\
\text { in PSZ relative to controls } \\
\text { - Controls showed gradual } \\
\text { increase in gamma during } \\
\text { maintenance, while PSZ } \\
\text { showed increased gamma } \\
\text { amplitudes pre- and } \\
\text { post-stimuli } \\
\text { - Controls' gamma responses } \\
\text { increased with WM load, } \\
\text { while PSZs' did not }\end{array}$ & $\begin{array}{l}\text { PSZ may need to inefficiently } \\
\text { initiate cognitive control on } \\
\text { simple tasks that controls } \\
\text { may complete via automated } \\
\text { processes }\end{array}$ \\
\hline
\end{tabular}


Table 3 Theta and gamma abnormalities in people with schizophrenia (PSZ) during working memory processes (Continued)

\begin{tabular}{|c|c|c|c|c|c|c|c|}
\hline $\begin{array}{l}\text { vii. Haenschel } \\
\text { et al. (2009) [29] }\end{array}$ & $\begin{array}{l}\text { - } 14 \text { patients with early } \\
\text { onset schizophrenia } \\
\text { (onset prior to age } \\
18 ; 5 \text { females, aged } \\
17.76 \pm 1.44 \text { years) } \\
-14 \text { controls (aged } \\
17.37 \pm 1.41 \text { years) }\end{array}$ & Scalp EEG & Scalp-wide & $\begin{array}{l}\text { Delayed visual } \\
\text { discrimination task }\end{array}$ & $\begin{array}{l}\text { Encoding, } \\
\text { maintenance and } \\
\text { retrieval }\end{array}$ & $\begin{array}{l}\text { - PSZ showed reductions in } \\
\text { induced gamma-power } \\
\text { during retrieval over both } \\
\text { anterior and posterior sites as } \\
\text { compared to controls } \\
\text { - Controls showed an increase } \\
\text { in induced gamma amplitudes } \\
\text { during late maintenance from } \\
\text { load } 1 \text { to } 2 \text { and a subsequent } \\
\text { decrease from } 2 \text { to 3; PSZ } \\
\text { showed the opposite pattern }\end{array}$ & $\begin{array}{l}\text { - PSZ may have difficulties } \\
\text { effectively retrieving stimulus } \\
\text { representations } \\
\text { - Differing patterns of } \\
\text { gamma modulation during } \\
\text { maintenance may reflect a } \\
\text { lower storage capacity limit } \\
\text { in PSZ as compared to controls }\end{array}$ \\
\hline $\begin{array}{l}\text { viii. Barr et al. } \\
\text { (2010) [118] }\end{array}$ & $\begin{array}{l}\text { - } 24 \text { participants with } \\
\text { schizophrenia }(n=19) \\
\text { or schizoaffective } \\
\text { disorder }(n=5) ; 10 \\
\text { females, aged } 37.09 \pm \\
11.04 \text { years) } \\
\text { - } 24 \text { healthy controls } \\
\text { (11 females, aged } \\
37.71 \pm 10.12 \text { years })\end{array}$ & Scalp EEG & $\begin{array}{l}\text { Scalp-wide; only } \\
\text { frontal electrodes } \\
\text { used for evoked } \\
\text { gamma analyses }\end{array}$ & Visual N-back task & Maintenance & $\begin{array}{l}\text { Increased evoked gamma-power } \\
\text { over frontal sites as well as } \\
\text { failure to modulate gamma-power } \\
\text { with increasing WM load in PSZ } \\
\text { as compared to healthy controls }\end{array}$ & $\begin{array}{l}\text { PSZ may allocate attentional } \\
\text { resources inefficiently }\end{array}$ \\
\hline $\begin{array}{l}\text { ix. Chen et al. } \\
\text { (2014) [119] }\end{array}$ & $\begin{array}{l}-12 \text { PSZ ( } 3 \text { females, } \\
\text { aged } 31 \pm 10.79 \text { years) } \\
-12 \text { controls ( } 6 \text { females, } \\
\text { aged } 33.08 \pm 8.23 \text { years) }\end{array}$ & Scalp EEG & $\begin{array}{l}\text { Scalp-wide; only } \\
\text { frontal electrodes } \\
\text { used for gamma- } \\
\text { analyses }\end{array}$ & $\begin{array}{l}\text { Modified Sternberg } \\
\text { paradigm }\end{array}$ & $\begin{array}{l}\text { Encoding, } \\
\text { maintenance, } \\
\text { retrieval }\end{array}$ & $\begin{array}{l}\text { - PSZ showed reduced frontal } \\
\text { (F3) gamma amplitudes during } \\
\text { all three WM stages as compared } \\
\text { to controls } \\
\text { - Gamma amplitudes correlated } \\
\text { with duration of illness in PSZ } \\
\text { - PSZ failed to modulate gamma } \\
\text { amplitudes across all WM stages } \\
\text { as compared to controls } \\
\text { (Supplementary materials) }\end{array}$ & $\begin{array}{l}\text { - PSZ demonstrate } \\
\text { impaired inter-regional } \\
\text { connections involving } \\
\text { ।the frontal lobe } \\
\text { - PSZ both lack and } \\
\text { misallocate cognitive } \\
\text { resources as } \\
\text { compared to controls }\end{array}$ \\
\hline
\end{tabular}


and/or first-episode patients), electrode site selection (e.g., single channel versus pooled sites) or means of collection (e.g., MEG vs. EEG). The literature nonetheless demonstrates consistent reports of abnormalities in theta- and gamma-band activity for PSZ as compared to healthy controls during WM processes, particularly in response to changes in task conditions related to WM function.

Though the present review focuses on the relation of theta- and gamma-oscillations to WM dysfunction in PSZ, the role of oscillatory activity in other frequencies and in relation to other cognitive functions has been reviewed elsewhere [28, 38, 66, 67]. Thus, abnormalities in oscillatory activity in PSZ do not appear to be limited to theta- and gamma-band activity, and may contribute to a wide variety of symptomatology observed in the disorder.

\section{Relevance of abnormalities in theta- and gamma- activity in people with schizophrenia}

PSZ demonstrate abnormalities in theta- and gamma- band activity during WM paradigms. Though no studies of WM in PSZ have examined coupling between thetaand gamma- activities, the ramifications of deficiencies within each of these bands independently on their interaction are easily ascertained. Inconsistencies in phase, as indexed by abnormal phase synchrony measures, would significantly interfere with any meaningful coordination between signals of differing frequencies. Similarly, amplitude deficiencies suggest abnormal generation of neural oscillations, be it through activation of fewer cells or impaired coordination of cellular assemblies that would function more cohesively in the healthy brain. Regardless, less reliable generators of oscillatory activity will limit the unitary functioning of cell assemblies that is necessary for coordination of signals that compose the theta-gamma neural code. Although coupling between thetaand gamma- activity in PSZ has been investigated, the few examinations of interactions between these bands in PSZ have not involved WM directly. Though theta-gamma coupling has been observed during processes other than WM in healthy people, the bulk of the literature ties it to memory processes; thus, further investigation is needed to clarify whether theta-gamma interactions are indeed abnormal during WM processes in PSZ, and whether those abnormalities explain behavioral performance deficits associated with the disorder.

\section{Conclusions: the theta-gamma neural code and visuospatial WM in schizophrenia}

Lisman and Idiart's [39] model positing a theta/gamma neural code as a means for representing multiple items in WM has generated a significant body of literature, and has been implicated in an even greater collection of research. A considerable amount of evidence has been accumulated and overwhelmingly supports the notions advanced by the model, namely that the interaction between theta- and gamma- oscillations communicate meaningful information within the brain in terms of memory and potentially other cognitive processes. Recent experimentation with humans has supported the model more directly: gamma activity associated with particular sequentially presented stimuli has been found to peak at different phases of concurrent theta activity in accordance with stimulus position within the sequence, and electrical stimulation in the gamma frequency range has shown to improve WM performance when administered at particular phases of theta. Regardless of how generalized the 
theta/gamma code is within the brain, it is difficult to dispute the notion that thetagamma interactions are central features of WM processes in humans and animals. Furthermore, measures of the strength of coupling between the two bands have repeatedly shown to predict WM performance, supporting the notion that theta-/gamma- interaction and memory are causally linked.

Despite this sizeable body of evidence supporting the importance of theta-gamma coupling to WM processes in humans and animals, few studies have examined interactions between the bands in PSZ [62, 63], and none in the context of WM. As such, much additional experimentation is required to determine whether deficient thetagamma coupling may explain WM deficits in PSZ. Notably, though several studies have demonstrated predictive relationships between measures of coupling and WM ability in healthy people, few have compared the predictive ability of theta-gamma coupling to other indices of oscillatory activity, including measures of power and/or phase synchrony within theta-, gamma-, and other frequency bands. One notable exception is [32], who found an index of alpha-band power predicted WM capacity better than indices of theta-gamma coupling; the researchers interpreted this alpha measure as an index of the suppression of irrelevant information, a mechanism arguably distinct from that being presently examined. Regardless, the logical question is thus whether deficient theta-gamma coupling truly explains WM deficiencies, or if it is merely an important correlate of some other primary cause. Addressing such a question is an important next step in further asserting the causal role of theta-gamma coupling in WM processes as well as the veracity of Lisman and Idiart's [39] theta/gamma neural code. ${ }^{2}$

Despite the unanswered questions that remain, considerable evidence has been found to support the theory of a theta/gamma code. Much of this evidence is derived from examinations of spatial WM processes in both rodents and humans. Given the well documented deficits in visuospatial WM in PSZ [6] and their relatives [9-11], a clear and testable hypothesis is that abnormal interactions between theta- and gammaactivity explains these deficits in visuospatial WM and WM at large. The hypothesis is supported by the considerable body of literature demonstrating abnormalities in thetaand gamma-bands individually within PSZ, including in connection with WM. Examination of theta-gamma coupling during WM in PSZ may yield a better understanding of mechanisms underlying the prominent WM deficits observed in people with the disorder. Lisman and Idiart's [39] theta-gamma neural code provides a framework for testing a specific source of WM dysfunction in PSZ. Using the Lisman and Idiart [39] model as a guide, we might expect to find generally reduced phase-phase coupling between theta- and gamma-activity during performance of tasks probing WM in PSZ. Theta-gamma coupling in healthy people has been observed prominently over prefrontal and posterior regions in scalp level recordings [32, 35, 36, 68-70]. Thus, deficits in synchrony between the two bands observed over anterior and posterior regions in scalp recordings, as well as in theta-gamma synchrony between the two areas, would particularly support the notion that deficits in the temporal alignment of theta- and gamma- activities are inextricably linked to WM function in the disorder. Furthermore, such synchrony deficits would mirror reports of inefficiencies in prefrontal areas [71] as well as deficient functional connectivity between prefrontal and posterior areas associated with WM processes observed in PSZ using functional MRI [72, 73], and may represent the same dysfunction. Theta-gamma interactions may 
likewise be disrupted in the hippocampus in PSZ, given the predominance of reported theta-gamma coupling during WM processes in animals and healthy people (e.g., Table 1) and observations that structural abnormalities in the hippocampus are prominent in schizophrenia [74] and observed even in cases of early-onset [75]. As activity from the hippocampus is difficult to observe using scalp level recordings (see [76]), intracranial EEG would be necessary to investigate hippocampal theta-gamma coupling deficits in PSZ; however, were such studies conducted, the Lisman and Idiart [39] model in combination with previous findings suggest abnormalities in thetagamma coupling would be likely.

Furthermore, investigations of theta-gamma abnormalities during spatial WM processing in PSZ would be more informative if they included a task with a sequential presentation of stimuli. The Lisman and Idiart [39] model predicts different thetaphase preferences for different stimuli, represented by individual gamma cycles superimposed upon a theta cycle, in a memory sequence. The discussed phenomenon of phase-precession observed in rodents $[50,51,56,58,59]$ represents one of the primary pieces of evidence supporting the theory and contributing to its continued consideration, and recent work has shown progressive shifts in gamma activity based on stimulus sequence [77]. However, paradigms that would allow for investigation of differential phase-preferences for individual stimuli in a spatial memory sequence are decidedly absent from human study. Thus, utilization of a paradigm featuring a sequential presentation of visuospatial stimuli would allow for a direct examination of whether phase-preferences are present in humans, and/or disrupted in PSZ.

In light of the dearth of literature testing the role of theta-gamma coupling in WM deficits in PSZ and their relatives, further study into these interactions between thetaand gamma-activity associated with WM in people genetically liable for schizophrenia is strongly warranted. Lisman and Idiart's [39] model provides a concrete framework for the design of an ideal investigation of these processes in PSZ, and suggests that deficits in theta-gamma interactions are likely to be observed in such experimentation. Better understanding of how neural oscillations factor into visuospatial WM deficits in the disorder could help inform interventions for improving memory performance, particularly in light of continually developing techniques of neuromodulation that have been shown to improve cognitive function using oscillatory stimulation (e.g., [78]).

\section{Endnotes}

${ }^{1}$ Sternberg himself recently reviewed the compatibility of Lisman and Idiart's [39] model with high-speed scanning research; see [120].

${ }^{2}$ Much of this paragraph is derived from insightful commentary from one of the paper's reviewers, which is greatly appreciated.

\section{Abbreviations}

PSZ: People with schizophrenia; REL: Unaffected first-degree biological relatives of people with schizophrenia; EEG: Electroencephalography; ERP: Event-related potential; ADP: Afterdepolarization; ICA: Independent component analysis; MRI: Magnetic resonance imaging; TGC: Theta-gamma coupling; iEEG: Intracranial EEG; LFP: Local field potential; CSD: Current source density; EC: Entorhinal cortex; HFO: High-frequency oscillation; ACC: Anterior cingulate cortex; PFC: Prefrontal cortex; MEG: Magnetoencephalography; WM: Working memory 


\section{Funding}

This work was supported by grants to Scott Sponheim from the National Institute of Mental Health (R03MH106831) and from the Veterans Health Administration Clinical Science Research and Development Program (ICX000227A).

\section{Availability of data and materials}

Data not shared: not applicable (review of existing literature).

\section{Authors' contributions}

PAL wrote the submitted manuscript; SRS advised writing of the manuscript and carried out editing of the submission. Both authors read and approved the final manuscript.

\section{Competing interests}

Both authors declare that they have no competing interests.

\section{Consent for publication}

Not applicable.

\section{Ethics approval and consent to participate}

Not applicable.

Received: 12 May 2016 Accepted: 26 October 2016

\section{Published online: 15 November 2016}

\section{References}

1. De Beni R, Pazzaglia F, Gyselinck V, Meneghetti C. Visuospatial working memory and mental representation of spatial descriptions. Eur J Cogn Psychol. 2005;17:77-95.

2. Meilinger $\mathrm{T}$, Knauff $\mathrm{M}, \mathrm{Bülthoff} \mathrm{HH}$. Working memory in wayfinding-A dual task experiment in a virtual city. Cognit Sci. 2008:32:755-70.

3. Anguera JA, Reuter-Lorenz PA, Willingham DT, Seidler RD. Contributions of spatial working memory to visuomotor learning. J Cogn Neurosci. 2010;22:1917-30.

4. Uresti-Cabrera LA, Diaz R, Vaca-Palomares I, Fernandez-Ruiz J. The effect of spatial working memory deterioration on strategic visuomotor learning across aging. Behav Neurol. 2015;2015:1-7.

5. Conklin HM, Curtis CE, Calkins ME, lacono WG. Working memory functioning in schizophrenia patients and their first-degree relatives: cognitive functioning shedding light on etiology. Neuropsychologia. 2005;43:930-42.

6. Lee J, Park S. Working memory impairments in schizophrenia: a meta-analysis. J Abnorm Psychol. 2005;114:599-611.

7. Goghari VM, Brett C, Tabraham P, Johns L, Valmaggia L, Broome M, Woolley J, Bramon E, Howes O, Byrne M, McGuire P. Spatial working memory ability in individuals at ultra high risk for psychosis. J Psychiatr Res. 2014;50:100-5

8. Takahashi H, Iwase M, Nakahachi T, Sekiyama R, Tabushi K, Kajimoto O, Shimizu A, Takeda M. Spatial working memory deficit correlates with disorganization symptoms and social functioning in schizophrenia. Psychiatry Clin Neurosci. 2005;59:453-60.

9. Park S, Holzman PS, Goldman-Rakic PS. Spatial working memory deficits in the relatives of schizophrenic patients. Arch Gen Psychiatry. 1995:52:821-8.

10. Pirkola T, Tuulio-Henriksson A, Glahn D, Kieseppä T, Haukka J, Kaprio J, Lönnqvist J, Cannon TD. Spatial working memory function in twins with schizophrenia and bipolar disorder. Biol Psychiatry. 2005;58:930-6.

11. Bachman P, Kim J, Yee CM, Therman S, Manninen M, Lönnqvist J, Kaprio J, Huttunen MO, Näätänen R, Cannon TD. Efficiency of working memory encoding in twins discordant for schizophrenia. Psychiatry Res. 2009;174:97-104.

12. Allen AJ, Griss ME, Folley BS, Hawkins KA, Pearlson GD. Endophenotypes in schizophrenia: a selective review. Schizophr Res. 2009;109:24-37.

13. Glahn DC, Therman S, Manninen M, Huttunen M, Kaprio J, Lönnqvist J, Cannon TD. Spatial working memory as an endophenotype for schizophrenia. Biol Psychiatry. 2003;53:624-6.

14. Gottesman II, Gould TD. The endophenotype concept in psychiatry: etymology and strategic intentions. Am J Psychiatry. 2003;160:636-45.

15. Snitz BE, MacDonald AW, Carter CS. Cognitive deficits in unaffected first-degree relatives of schizophrenia patients: a meta-analytic review of putative endophenotypes. Schizophr Bull. 2006;32:179-94.

16. Perez VB, Vogel EK, Luck S, Kappenman E. What ERPs can tell us about working memory, The Oxford Handbook of Event-Related Potential Components. 2012. p. 361-72.

17. Dias EC, Butler PD, Hoptman MJ, Javitt DC. Early sensory contributions to contextual encoding deficits in schizophrenia. Arch Gen Psychiatry. 2011;68:654-64.

18. Haenschel C, Bittner RA, Haertling F, Rotarska-Jagiela A, Maurer K, Singer W, Linden DE. Contribution of impaired early-stage visual processing to working memory dysfunction in adolescents with schizophrenia: a study with event-related potentials and functional magnetic resonance imaging. Arch Gen Psychiatry. 2007;64:1229-40.

19. Zhao YL, Tan SP, De Yang F, Wang LL, Feng WF, Chan RC, Gao X, Zhou DF, Li BB, Song CS, et al. Dysfunction in different phases of working memory in schizophrenia: evidence from ERP recordings. Schizophr Res. 2011;133:112-9.

20. Davenport ND, Sponheim SR, Stanwyck JJ. Neural anomalies during visual search in schizophrenia patients and unaffected siblings of schizophrenia patients. Schizophr Res. 2006;82:15-26.

21. Ergen M, Marbach S, Brand A, Başar-Eroğlu C, Demiralp T. P3 and delta band responses in visual oddball paradigm in schizophrenia. Neurosci Lett. 2008;440:304-8.

22. Lee SY, Namkoong K, Cho HH, Song D-H, An SK. Reduced visual P300 amplitudes in individuals at ultra-high risk for psychosis and first-episode schizophrenia. Neurosci Lett. 2010;486:156-60. 
23. Oribe N, Hirano Y, Kanba S, del Re EC, Seidman LJ, Mesholam-Gately R, Spencer KM, McCarley RW, Niznikiewicz MA. Early and late stages of visual processing in individuals in prodromal state and first episode schizophrenia: an ERP study. Schizophr Res. 2013;146:95-102

24. Yeap S, Kelly SP, Sehatpour P, Magno E, Garavan H, Thakore JH, Foxe JJ. Visual sensory processing deficits in Schizophrenia and their relationship to disease state. Eur Arch Psychiatry Clin Neurosci. 2008; 258:305-16.

25. Sponheim SR, McGuire KA, Stanwyck JJ. Neural anomalies during sustained attention in first-degree biological relatives of schizophrenia patients. Biol Psychiatry. 2006;60:242-52.

26. Yeap S, Kelly SP, Sehatpour P, Magno E, Javitt DC, Garavan H, Thakore JH, Foxe JJ. Early visual sensory deficits as endophenotypes for schizophrenia. Arch Gen Psychiatry. 2006;63:1180-8.

27. Spellman TJ, Gordon JA. Synchrony in schizophrenia: a window into circuit-level pathophysiology. Curr Opin Neurobiol. 2015;30:17-23.

28. Uhlhaas PJ, Singer W. Abnormal neural oscillations and synchrony in schizophrenia. Nat Rev Neurosci. 2010;1 1:100-13

29. Haenschel C, Bittner RA, Waltz J, Haertling F, Wibral M, Singer W, Linden DE, Rodriguez E. Cortical oscillatory activity is critical for working memory as revealed by deficits in early-onset schizophrenia. J Neurosci. 2009;29:9481-9.

30. Nyhus E, Curran T. Functional role of gamma and theta oscillations in episodic memory. Neurosci Biobehav Rev. 2010:34:1023-35.

31. Lisman JE, Jensen O. The theta-gamma neural code. Neuron. 2013;77:1002-16.

32. Sauseng P, Klimesch W, Heise KF, Gruber WR, Holz E, Karim AA, Glennon M, Gerloff C, Birbaumer N, Hummel FC. Brain oscillatory substrates of visual short-term memory capacity. Curr Biol. 2009;19:1846-52.

33. Axmacher $\mathrm{N}$, Henseler MM, Jensen $\mathrm{O}$, Weinreich I, Elger CE, Fell J. Cross-frequency coupling supports multi-item working memory in the human hippocampus. Proc Natl Acad Sci. 2010;107:3228-33.

34. Chaieb L, Leszczynski M, Axmacher N, Höhne M, Elger CE, Fell J. Theta-gamma phase-phase coupling during working memory maintenance in the human hippocampus. Cogn Neurosci. 2015;6:149-57.

35. Park JY, Jhung K, Lee J, An SK. Theta-gamma coupling during a working memory task as compared to a simple vigilance task. Neurosci Lett. 2013;532:39-43.

36. Park JY, Lee Y-R, Lee J. The relationship between theta-gamma coupling and spatial memory ability in older adults. Neurosci Lett. 2011:498:37-41.

37. Lisman JE, Buzsáki G. A neural coding scheme formed by the combined function of gamma and theta oscillations. Schizophr Bull. 2008;34:974-80.

38. Moran LV, Hong LE. High vs low frequency neural oscillations in schizophrenia. Schizophr Bull. 2011;37:659-63.

39. Lisman JE, Idiart MA. Storage of 7+/-2 short-term memories in oscillatory subcycles. Science. 1995;267:1512-5.

40. Koene RA, Hasselmo ME. First-in-first-out item replacement in a model of short-term memory based on persistent spiking. Cereb Cortex. 2007;17:1766-81.

41. Bland BH. The physiology and pharmacology of hippocampal formation theta rhythms. Prog Neurobiol. 1986;26:1-54.

42. Steriade M, Dossi RC, Pare D, Oakson G. Fast oscillations $(20-40 \mathrm{~Hz})$ in thalamocortical systems and their potentiation by mesopontine cholinergic nuclei in the cat. Proc Natl Acad Sci. 1991;88:4396-400.

43. Andrade R. Cell excitation enhances muscarinic cholinergic responses in rat association cortex. Brain Res. 1991;548:81-93.

44. Caeser M, Brown DA, Gähwiler BH, Knöpfel T. Characterization of a calcium-dependent current generating a slow afterdepolarization of CA3 pyramidal cells in Rat hippocampal slice cultures. Eur J Neurosci. 1993;5:560-9.

45. Storm JF. An after-hyperpolarization of medium duration in rat hippocampal pyramidal cells. J Physiol. 1989:409:171-90.

46. Sternberg S. High-speed scanning in human memory. Science. 1966;153:652-4.

47. Jensen O, Lisman JE. An oscillatory short-term memory buffer model can account for data on the Sternberg task. J Neurosci. 1998;18:10688-99.

48. Sternberg S. Estimating the Distribution of Additive Reaction-Time Components.0

49. Miller GA. The magical number seven, plus or minus two: some limits on our capacity for processing information. Psychol Rev. 1956;63:81-97.

50. O'Keefe J, Recce ML. Phase relationship between hippocampal place units and the EEG theta rhythm. Hippocampus. 1993;3:317-30.

51. Skaggs WE, McNaughton BL, Wilson MA, Barnes CA. Theta phase precession in hippocampal neuronal populations and the compression of temporal sequences. Hippocampus. 1996;6:149-72.

52. Fox S, Wolfson S, Ranck Jr J. Hippocampal theta rhythm and the firing of neurons in walking and urethane anesthetized rats. Exp Brain Res. 1986;62:495-508.

53. Buzsáki G, Leung L-WS, Vanderwolf CH. Cellular bases of hippocampal EEG in the behaving rat. Brain Res Rev. 1983;6:139-71.

54. Sinclair BR, Seto MG, Bland BH. Theta-cells in $\mathrm{CA} 1$ and dentate layers of hippocampal formation: relations to slow-wave activity and motor behavior in the freely moving rabbit. J Neurophysiol. 1982;48:1214-25.

55. Otto T, Eichenbaum H, Wible CG, Wiener SI. Learning-related patterns of CA1 spike trains parallel stimulation parameters optimal for inducing hippocampal long-term potentiation. Hippocampus. 1991;1:181-92.

56. Lisman JE, Redish AD. Prediction, sequences and the hippocampus. Philos Trans R Soc Lond B Biol Sci. 2009;364:1193-201.

57. Schomburg EW, Fernández-Ruiz A, Mizuseki K, Berényi A, Anastassiou CA, Koch C, Buzsáki G. Theta phase segregation of input-specific gamma patterns in entorhinal-hippocampal networks. Neuron. 2014;84:470-85.

58. Bieri KW, Bobbitt KN, Colgin LL. Slow and fast gamma rhythms coordinate different spatial coding modes in hippocampal place cells. Neuron. 2014;82:670-81.

59. De Almeida L, Idiart M, Villavicencio A, Lisman J. Alternating predictive and short-term memory modes of entorhinal grid cells. Hippocampus. 2012;22:1647-51.

60. Belluscio MA, Mizuseki K, Schmidt R, Kempter R, Buzsáki G. Cross-frequency phase-phase coupling between theta and gamma oscillations in the hippocampus. J Neurosci. 2012;32:423-35.

61. Canolty RT, Edwards E, Dalal SS, Soltani M, Nagarajan SS, Kirsch HE, Berger MS, Barbaro NM, Knight RT. High gamma power is phase-locked to theta oscillations in human neocortex. Science. 2006;313:1626-8. 
62. Allen EA, Liu J, Kiehl KA, Gelernter J, Pearlson GD, Perrone-Bizzozero NI, Calhoun VD. Components of cross-frequency modulation in health and disease. Front Syst Neurosci. 2011;5:1-16.

63. Kirihara K, Rissling AJ, Swerdlow NR, Braff DL, Light GA. Hierarchical organization of gamma and theta oscillatory dynamics in schizophrenia. Biol Psychiatry. 2012;71:873-80.

64. Neymotin SA, Lazarewicz MT, Sherif M, Contreras D, Finkel LH, Lytton WW. Ketamine disrupts theta modulation of gamma in a computer model of hippocampus. J Neurosci. 2011;31:11733-43.

65. Caixeta FV, Cornélio AM, Scheffer-Teixeira R, Ribeiro S, Tort AB. Ketamine alters oscillatory coupling in the hippocampus. Sci Rep. 2013;3:1-10.

66. Senkowski D, Gallinat J. Dysfunctional Prefrontal Gamma-band Oscillations Reflect Working Memory and Other Cognitive Deficits in Schizophrenia. Biol Psychiatry. 2015;77:1010-19.

67. Pittman-Polletta BR, Kocsis B, Vijayan S, Whittington MA, Kopell NJ. Brain Rhythms Connect Impaired Inhibition to Altered Cognition in Schizophrenia. Biol Psychiatry. 2015;77:1020-30.

68. Holz EM, Glennon M, Prendergast K, Sauseng P. Theta-gamma phase synchronization during memory matching in visual working memory. Neuroimage. 2010;52:326-35.

69. Sauseng P, Klimesch W, Gruber WR, Birbaumer N. Cross-frequency phase synchronization: a brain mechanism of memory matching and attention. Neuroimage. 2008;40:308-17.

70. Schack B, Vath N, Petsche H, Geissler H-G, Möller E. Phase-coupling of theta-gamma EEG rhythms during short-term memory processing. Int J Psychophysiol. 2002;44:143-63.

71. Potkin S, Turner J, Brown G, McCarthy G, Greve D, Glover G, Manoach D, Belger A, Diaz M, Wible C, Ford J, Mathalon D, Gollub R, Lauriello J, O'Leary D, van Erp T, Toga A, Preda A, Lim K. FBIRN: working memory and DLPFC inefficiency in schizophrenia: the FBIRN study. Schizophr Bull. 2009;35:19-31.

72. Kang SS, Sponheim SR, Chafee MV, MacDonald AW. Disrupted functional connectivity for controlled visual processing as a basis for impaired spatial working memory in schizophrenia. Neuropsychologia. 2011;49:2836-47.

73. Poppe AB, Carter CS, Minzenberg MJ, MacDonald AW. Task-based functional connectivity as an indicator of genetic liability to schizophrenia. Schizophr Res. 2015;162:118-23.

74. MacDonald AW, Schulz SC. What we know: findings that every theory of schizophrenia should explain. Schizophr Bull. 2009:35:493-508.

75. White T, Cullen K, Rohrer LM, Karatekin C, Luciana M, Schmidt M, Hongwanishkul D, Kumra S, Schulz SC, Lim KO. Limbic structures and networks in children and adolescents with schizophrenia. Schizophr Bull. 2008;34:18-29.

76. Mormann F, Fell J, Axmacher N, Weber B, Lehnertz K, Elger CE, Fernández G. Phase/amplitude reset and theta-gamma interaction in the human medial temporal lobe during a continuous word recognition memory task. Hippocampus. 2005;15:890-900.

77. Heusser AC, Poeppel D, Ezzyat Y, Davachi L. Episodic sequence memory is supported by a theta-gamma phase code. Nat Neurosci. 2016;19:1374-80.

78. Lee DJ, Gurkoff GG, Izadi A, Berman RF, Ekstrom AD, Muizelaar JP, Lyeth BG, Shahlaie K. Medial septal nucleus theta frequency deep brain stimulation improves spatial working memory after traumatic brain injury. J Neurotrauma. 2013;30:131-9.

79. Fell J, Axmacher $\mathrm{N}$. The role of phase synchronization in memory processes. Nat Rev Neurosci. 2011;12:105-18.

80. Tort AB, Kramer MA, Thorn C, Gibson DJ, Kubota Y, Graybiel AM, Kopell NJ. Dynamic cross-frequency couplings of local field potential oscillations in rat striatum and hippocampus during performance of a T-maze task. Proc Natl Acad Sci. 2008;105:20517-22.

81. Tort AB, Komorowski RW, Manns JR, Kopell NJ, Eichenbaum H. Theta-gamma coupling increases during the learning of item-context associations. Proc Natl Acad Sci. 2009;106:20942-7.

82. Shirvalkar PR, Rapp PR, Shapiro ML. Bidirectional changes to hippocampal theta-gamma comodulation predict memory for recent spatial episodes. Proc Natl Acad Sci. 2010;107:7054-9.

83. Cabral HO, Vinck M, Fouquet C, Pennartz CM, Rondi-Reig L, Battaglia FP. Oscillatory dynamics and place field maps reflect hippocampal ensemble processing of sequence and place memory under NMDA receptor control. Neuron. 2014;81:402-15.

84. Igarashi KM, Lu L, Colgin LL, Moser M-B, Moser El. Coordination of entorhinal-hippocampal ensemble activity during associative learning. Nature. 2014;510:143-7.

85. Nishida H, Takahashi M, Lauwereyns J. Within-session dynamics of theta-gamma coupling and high-frequency oscillations during spatial alternation in rat hippocampal area CA1. Cogn Neurodynamics. 2014;8:363-72.

86. Takahashi M, Nishida H, Redish AD, Lauwereyns J. Theta phase shift in spike timing and modulation of gamma oscillation: a dynamic code for spatial alternation during fixation in rat hippocampal area CA1. J Neurophysiol. 2014;111:1601-14.

87. Trimper JB, Stefanescu RA, Manns JR. Recognition memory and theta-gamma interactions in the hippocampus. Hippocampus. 2014;24:341-53.

88. Siegle JH, Wilson MA. Enhancement of encoding and retrieval functions through theta phase-specific manipulation of hippocampus. eLife. 2014;3:1-18.

89. Soltesz I, Deschenes M. Low-and high-frequency membrane potential oscillations during theta activity in CA1 and CA3 pyramidal neurons of the rat hippocampus under ketamine-xylazine anesthesia. J Neurophysiol. 1993;70:97-116.

90. Bragin A, Jandó G, Nádasdy Z, Hetke J, Wise K, Buzsáki G. Gamma (40-100 Hz) oscillation in the hippocampus of the behaving rat. J Neurosci. 1995;15:47-60.

91. Chrobak JJ, Buzsáki G. Gamma oscillations in the entorhinal cortex of the freely behaving rat. J Neurosci. 1998;18:388-98.

92. Buzsáki G, Buhl D, Harris K, Csicsvari J, Czeh B, Morozov A. Hippocampal network patterns of activity in the mouse. Neuroscience. 2003;116:201-11.

93. Csicsvari J, Jamieson B, Wise KD, Buzsáki G. Mechanisms of gamma oscillations in the hippocampus of the behaving rat. Neuron. 2003;37:311-22.

94. Hentschke H, Perkins MG, Pearce RA, Banks MI. Muscarinic blockade weakens interaction of gamma with theta rhythms in mouse hippocampus. Eur J Neurosci. 2007;26:1642-56. 
95. Sirota A, Montgomery S, Fujisawa S, Isomura Y, Zugaro M, Buzsáki G. Entrainment of neocortical neurons and gamma oscillations by the hippocampal theta rhythm. Neuron. 2008;60:683-97.

96. Wulff P, Ponomarenko AA, Bartos M, Korotkova TM, Fuchs EC, Bähner F, Both M, Tort AB, Kopell NJ, Wisden W, Monyer $\mathrm{H}$. Hippocampal theta rhythm and its coupling with gamma oscillations require fast inhibition onto parvalbumin-positive interneurons. Proc Natl Acad Sci. 2009;106:3561-6.

97. Quilichini P, Sirota A, Buzsáki G. Intrinsic circuit organization and theta-gamma oscillation dynamics in the entorhinal cortex of the rat. J Neurosci. 2010;30:11128-42.

98. Newman EL, Gillet SN, Climer JR, Hasselmo ME. Cholinergic blockade reduces theta-gamma phase amplitude coupling and speed modulation of theta frequency consistent with behavioral effects on encoding. J Neurosci. 2013;33:19635-46.

99. Pernía-Andrade AJ, Jonas P. Theta-gamma-modulated synaptic currents in hippocampal granule cells in vivo define a mechanism for network oscillations. Neuron. 2014;81:140-52.

100. Yamamoto J, Suh J, Takeuchi D, Tonegawa S. Successful execution of working memory linked to synchronized high-frequency gamma oscillations. Cell. 2014;157:845-57.

101. Cunningham MO, Davies CH, Buhl EH, Kopell N, Whittington MA. Gamma oscillations induced by kainate receptor activation in the entorhinal cortex in vitro. J Neurosci. 2003;23:9761-9.

102. Goutagny R, Gu N, Cavanagh C, Jackson J, Chabot J-G, Quirion R, Krantic S, Williams S. Alterations in hippocampal network oscillations and theta-gamma coupling arise before A-beta overproduction in a mouse model of Alzheimer's disease. Eur J Neurosci. 2013;37:1896-902.

103. Pastoll H, Solanka L, van Rossum MC, Nolan MF. Feedback inhibition enables theta-nested gamma oscillations and grid firing fields. Neuron. 2013;77:141-54

104. Lakatos P, Shah AS, Knuth KH, Ulbert I, Karmos G, Schroeder CE. An oscillatory hierarchy controlling neuronal excitability and stimulus processing in the auditory cortex. J Neurophysiol. 2005;94:1904-11.

105. Voloh B, Valiante TA, Everling S, Womelsdorf T. Theta-gamma coordination between anterior cingulate and prefrontal cortex indexes correct attention shifts. Proc Natl Acad Sci. 2015;112:8457-62.

106. Fell J, Klaver P, Elfadil H, Schaller C, Elger CE, Fernández G. Rhinal-hippocampal theta coherence during declarative memory formation: interaction with gamma synchronization? Eur J Neurosci. 2003;17:1082-8.

107. Van der Meij R, Kahana M, Maris E. Phase-amplitude coupling in human electrocorticography is spatially distributed and phase diverse. J Neurosci. 2012;32:111-23.

108. Maris $E$, van Vugt M, Kahana M. Spatially distributed patterns of oscillatory coupling between high-frequency amplitudes and low-frequency phases in human iEEG. Neuroimage. 2011;54:836-50.

109. Schack B, Weiss S. Quantification of phase synchronization phenomena and their importance for verbal memory processes. Biol Cybern. 2005;92:275-87.

110. Lee $Y-Y$, Yang $C-Y$. Utilizing the extent of theta-gamma synchronization to estimate visuospatial memory ability. Australas Phys Eng Sci Med. 2014;37:665-72.

111. Vosskuhl J, Huster RJ, Herrmann CS. Increase in short-term memory capacity induced by down-regulating individual theta frequency via transcranial alternating current stimulation. Front Hum Neurosci. 2015;9:257.

112. Alekseichuk I, Turi Z, de Lara GA, Antal A, Paulus W. Spatial Working Memory in Humans Depends on Theta and High Gamma Synchronization in the Prefrontal Cortex. Curr Biol. 2016;26:1513-21.

113. Schmiedt C, Brand A, Hildebrandt H, Başar-Eroğlu C. Event-related theta oscillations during working memory tasks in patients with schizophrenia and healthy controls. Cogn Brain Res. 2005;25:936-47.

114. Missonnier P, Herrmann FR, Zanello A, Bâ MB, Curtis L, Canovas D, Chantraine F, Richiardi J, Giannakopoulos P, Merlo MC. Event-related potentials and changes of brain rhythm oscillations during working memory activation in patients with first-episode psychosis. J Psychiatry Neurosci. 2012;37:95.

115. Griesmayr B, Berger B, Stelzig-Schoeler R, Aichhorn W, Bergmann J, Sauseng P. EEG theta phase coupling during executive control of visual working memory investigated in individuals with schizophrenia and in healthy controls. Cogn Affect Behav Neurosci. 2014;14:1340-55.

116. Kissler J, Müller MM, Fehr T, Rockstroh B, Elbert T. MEG gamma band activity in schizophrenia patients and healthy subjects in a mental arithmetic task and at rest. Clin Neurophysiol. 2000;111:2079-87.

117. Başar-Eroğlu C, Brand A, Hildebrandt H, Kedzior KK, Mathes B, Schmiedt C. Working memory related gamma oscillations in schizophrenia patients. Int J Psychophysiol. 2007;64:39-45.

118. Barr M, Farzan F, Tran LC, Chen R, Fitzgerald P, Daskalakis Z. Evidence for excessive frontal evoked gamma oscillatory activity in schizophrenia during working memory. Schizophr Res. 2010;121:146-52.

119. Chen C-MA, Stanford AD, Mao X, Abi-Dargham A, Shungu DC, Lisanby SH, Schroeder CE, Kegeles LS. GABA level, gamma oscillation, and working memory performance in schizophrenia. Neurolmage. 2014;4:531-9.

120. Sternberg S. In defence of high-speed memory scanning. Q J Exp Psychol. 2016;69:2020-75. 